\title{
MHD HEAT TRANSFER IN TWO-LAYERED FLOW OF CONDUCTING FLUIDS THROUGH A CHANNEL BOUNDED BY TWO PARALLEL POROUS PLATES IN A ROTATING SYSTEM
}

\author{
T. LINGA RAJU* \\ Department of Engineering Mathematics \\ AUCE(A), Andhra University \\ VISAKHAPATNAM, Pin code: 530 003, A.P, INDIA \\ E-mail: tlraju45@yahoo.com \\ B. NEELA RAO \\ Department of Mathematics \\ Aditya Institute of Technology and Management \\ TEKKALI, Pin code: 532 201, A.P, INDIA \\ E-mail: neelarao.b@gmail.com
}

\begin{abstract}
The paper aims to analyze the heat transfer aspects of a two-layered fluid flow in a horizontal channel under the action of an applied magnetic and electric fields, when the whole system is rotated about an axis perpendicular to the flow. The flow is driven by a common constant pressure gradient in the channel bounded by two parallel porous insulating plates, one being stationary and the other one oscillatory. The fluids in the two regions are considered electrically conducting, and are assumed to be incompressible with variable properties, namely, different densities, viscosities, thermal and electrical conductivities. The transport properties of the two fluids are taken to be constant and the bounding plates are maintained at constant and equal temperature. The governing partial differential equations are then reduced to the ordinary linear differential equations by using a two-term series. The temperature distributions in both fluid regions of the channel are derived analytically. The results are presented graphically to discuss the effect on the heat transfer characteristics and their dependence on the governing parameters, i.e., the Hartmann number, Taylor number, porous parameter, and ratios of the viscosities, heights, electrical and thermal conductivities. It is observed that, as the Coriolis forces become stronger, i.e., as the Taylor number increases, the temperature decreases in the two fluid regions. It is also seen that an increase in porous parameter diminishes the temperature distribution in both the regions.
\end{abstract}

Key words: MHD, two-layered fluids/immiscible fluids, unsteady flow, heat transfer, rigid rotation, porous boundaries.

\section{Introduction}

Many investigations on the problems of a magnetohydrodynamic (MHD) fluid system contain the single fluid motions through parallel plate / rectangular channels with or without considering the rigid rotation/suction-injection and under steady or unsteady motions have been reported in the literature by several investigators since long due to their importance in various engineering, technological and industrial applications. But most of the problems relating to astrophysics, geophysical fluid dynamics, aeronautics, in petroleum industry and in industrial applications etc; involve multi-layered-fluid flow situations. Often, in the petroleum industry as well as in engineering and technological fields, a stratified two-phase or twolayered fluid flow occurs. Transportation and extraction of the products of oil are other obvious applications using a two-phase/two-layered fluid flow system to obtain the increased flow rates in an electromagnetic

\footnotetext{
* To whom correspondence should be addressed
} 
pump with the possibility of reducing the power required to pump oil in a pipe line by a suitable addition of water (Shail [1]). Considerable progress has been made in the research studies of two-layered fluid flows by several authors, notable amongst them are Walin [2], Packham and Shail [3], Lielausis [4], Debnath and Basu [5], Michiyoshi et al. [6], Dunn [7], Gherson [8], Lohrasbi and Sahai [9], Alireza and Sahai [10], Serizawa et al. [11], Malashetty and Leela [12], Ramadan and Chamkha [13], Chamkha [14], Raju and Murty [15], Tsuyoshi and Shu-Ichiro [16] etc. The MHD heat transfer processes are of interest in power engineering, metallurgy, astrophysics, geophysics and in petroleum industry. Other applications include transport phenomena in power transform electronics, heating and cooling processes in semi-conductor electronics, absorption reactors and in aerodynamic heating. Moreover, the magnetohydrodynamic (MHD) flows can also be treated as a viable option for transporting conducting fluids in microscale systems; for instance, the flow inside the micro-channel networks of a lab-on-a-chip device (Haim et al. [17], Hussameddine et al. [18]. Multiple fluids in micro-fluidic devices can be transported through a channel for different reasons. For example, an increase in mobility of a fluid may be achieved by stratification of a highly mobile fluid or mixing of two or more fluids in transit may be designed for emulsification or heat and mass transfer applications. In this regard, magnetic field-driven micro-pumps are an increasing demand due to their long-term reliability in generating flow, low power requirement and in mixing efficiency (as in Yi et al. [19]; Weston et al. [20]). The phenomenon of rotation is always encountered and is very often observed in cosmological and geophysical sciences (Hide and Roberts [21], Greenspan [22] and Diek [23]). The rotating flow of an electrically conducting fluid in the presence of a magnetic field is encountered in geophysical fluid dynamics. It is also important in the solar physics dealing with the sunspot development (Elco et al. [24], Katsurai [25], Kolesnikov and Khait [26], Yantovskry and Tolmach [27]). Also, rotating heat exchangers are extensively used by the chemical and automobile industries. Many authors have focused on the theoretical/experimental investigation of hydromagnetic flows in a rotating environment, because of their occurrence in several natural phenomena that are directly governed by the actions of the Coriolis forces and their application in various technological situations. An order of magnitude analysis shows that in basic flow field equations the effects of the Coriolis force is more significant as compared to that of inertia and viscous forces. It is worth noting that the Coriolis and magnetohydrodynamic forces are comparable in magnitude and these Coriolis forces induce a secondary flow in the fluid (Holton [28], Batchelor [29] and Gupta [30]). Considerable attention has been also given to the study of an unsteady magnetohydrodynamic flow, heat transfer and their response due to the imposed oscillations/impulsive motion of a boundary or boundary temperature under the presence of an external magnetic field (Tao [31], Gupta [32], Stanisic et al. [33], Katagiri [34], Nanda and Mahanty [35], Debnath [36], Jana et al. [37], Seth et al. [38], Ghosh [39], Chandran et al. [40], Ghosh and Bhatttacharee [41], Singh [42], Hayat et al. [43], Guria [44]). Despite these studies, the effects of unsteady two-layered fluid flows through horizontal channels received much less attention in the literature. The unsteady hydromagnetic flow of electrically conducting two-layered fluid flow in a rotating environment over the porous boundaries gained significant theoretical and practical importance owing to their applications in the petroleum industry, geophysical fluid dynamics, plasma physics, magnetohydrodynamics and in many such areas involving multi-layered-fluid flow situations. These flows seem to be important and play interesting roles in the flow pattern as most of the practical problems dealing with immiscible fluids are unsteady in nature. Also in many practical problems, obviously it is advantageous to consider both immiscible fluids as electrically conducting; where one of which is highly electrically conducting compared to the other. The fluid of low electrical conductivity compared to the other is functional to reduce the power required to pump the fluid in MHD pumps and flow meters. In view of these facts, Chamkha [45] studied an unsteady MHD convective heat and mass transfer past a semi-infinite vertical permeable moving plate with heat absorption. Umavathi et al. [46] investigated oscillatory Hartmann twofluid flow and heat transfer in a horizontal channel. Raju and Sreedhar [47] discussed usteady two-fluid flow and heat transfer of conducting fluids in channels under transverse magnetic field. Raju and Valli [48] discussed an unsteady two-layered fluid flow and heat transfer of conducting fluids in a channel between parallel porous plates under transverse magnetic field. Again in 2014, these authors have studied MHD twolayered unsteady fluid flow and heat transfer through a horizontal channel between parallel plates in a 
rotating system. Consequently, Raju and Rao [49] studied an unsteady two-layered fluid flow of conducting fluids in a channel between parallel porous plates under transverse magnetic field in a rotating system.

In the present paper, the magnetohydrodynamic (MHD) heat transfer in a two-layered flow of conducting fluids through a horizontal channel bounded by two parallel porous plates in a rotating system in presence of an applied magnetic and electric fields is investigated theoretically. It is assumed that, the fluids and the plates are in a state of rigid rotation with an angular velocity about an axis normal to the flow. The flow is driven by a constant common pressure gradient in the channel bounded by two parallel porous insulating plates, one being stationary and the other an oscillatory nature. The fluids in the two regions are considered as electrically conducting and are assumed to be incompressible with variable properties, namely, different densities, viscosities, thermal and electrical conductivities. The transport properties of the two fluids are considered to be constant and the bounding plates are maintained at constant and equal temperature. The resulting governing partial differential equations are then reduced to linear ordinary differential equations by using a two-term series. Analytical solutions for temperature distributions are obtained in both fluid regions of the channel. Profiles of these solutions are plotted to discuss the effect on the temperature fields and their dependence on the governing parameters involved, such as the Hartmann number, Taylor number (rotation parameter), suction number (porous parameter), ratio of the viscosities, heights and electrical conductivities. Also an examination is made how the temperature distributions vary through hydromagnetic interaction in the case of steady and unsteady motions with rigid rotation over the porous boundaries.

The results of this study are expected to be useful in understanding the effect of the presence of a slag layer on heat transfer characteristics of a coal fired rotating MHD generator, flow meters, in nuclear reactor and in space craft technology, etc. Also, such flows are encountered in many industrial applications, such as in liquid metals, metal working process, geothermal energy extracts and many other applications. It is also important to understand the dynamics of interfaces between the fluids and its effect on the transport characteristics of the system.

The structure of the paper is as follows. An introduction to the problem is given in section 1 . The formulation and mathematical analysis of the problem for equations of motion, the boundary and interface conditions are presented in section 2. In section 3, solutions of the problem are given. While section 4 gives the results and discussion based on the temperature profiles (which are displayed in Figs 2 to 8 ). And the conclusion is given in section 5 , followed by nomenclature and references.

\section{Formulation and mathematical analysis for the governing equations of motion, energy, boundary and interface conditions}

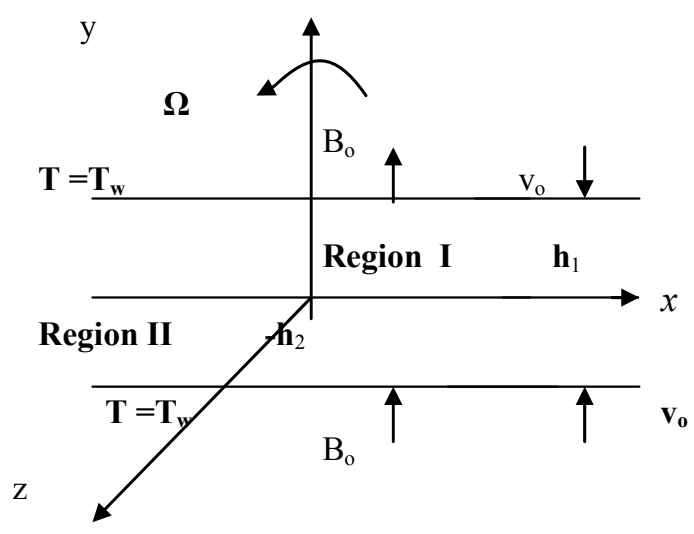

Fig.1. flow model and co-ordinate system. 
An unsteady magnetohydrodynamic (MHD) two layered-fluid flow in a horizontal channel consisting of two infinite parallel porous plates with constant suction $\mathrm{v}_{0}$ normal to these plates extending along the $x$ - and $z$-directions at $y=h_{1}$ and $y=-h_{2}$ is considered. The whole system is rotated with an angular velocity $\Omega$ in a counter clockwise direction about the $y$-axis perpendicular to the plates. The fluids in the upper and lower regions, that is $0 \leq y \leq h_{1}$ and $-h_{2} \leq y \leq 0$, are designated as Region -I and Region-II respectively. In the usual rectangular Cartesian coordinate system, the $x$-axis is taken in the direction of hydrodynamic pressure gradient in the plane parallel to the channel plates, but not in the direction of flow and $y$-axis at right angles to it. The length of the plates is considered much larger than the distance between them. Figure 1 depicts the flow model and co-ordinate system choosing the origin midway between the two plates. The flow in both upper and lower regions is driven by a common constant pressure gradient $\left(-\frac{\partial p}{\partial x}\right)$ in a channel bounded by two parallel porous plates, one being stationary and the other one oscillatory. The two bounding plates are maintained at constant temperature $\mathrm{T}_{W}\left(=\mathrm{T}_{W_{1}}=\mathrm{T}_{W_{2}}\right)$. A magnetic field of uniform strength $B_{0}$ is assumed to be applied transversely to the direction of flow, that is, along the $y$ direction. A constant electric field $E_{0}$ is also applied in the $z$ - direction. The induced magnetic field is being neglected by assuming that it is small when compared to an applied field. Both the regions are occupied by two immiscible electrically conducting, incompressible fluids with different densities $\rho_{l}, \rho_{2}$, viscosities $\mu_{1}, \mu_{2}$, electrical conductivities $\sigma_{1}, \sigma_{2}$ and thermal conductivities $K_{1}, K_{2}$. The plates are infinite in extent and hence all the physical variables except the pressure gradient are functions of y and t only. With these assumptions, the non-dimensional governing equations of motion, current, energy, the corresponding boundary and interface conditions as in Lohrasbi and Sahai [9] for an unsteady MHD flows in both fluid regions in a rotating frame of reference (Batchelor [29]) are expressed as

\section{Region-I:}

$$
\begin{aligned}
& \rho_{1} \frac{\partial u_{1}}{\partial t}-\mu_{1} \frac{\partial^{2} u_{1}}{\partial y^{2}}+\rho_{1} v_{0} \frac{\partial u_{1}}{\partial y}+\frac{\partial p}{\partial x}+\sigma_{1} u_{1} B_{0}^{2}+\sigma_{1} E_{0} B_{0}=-2 \rho_{1} \Omega w_{1}, \\
& \rho_{1} \frac{\partial w_{1}}{\partial t}-\mu_{1} \frac{\partial^{2} w_{1}}{\partial y^{2}}+\rho_{1} v_{0} \frac{\partial w_{1}}{\partial y}+\sigma_{1} u_{1} B_{0}^{2}=2 \rho_{1} \Omega u_{1}, \\
& \rho_{1} c_{p_{1}} \frac{\partial T_{1}}{\partial t}-K_{1} \frac{\partial^{2} T_{1}}{\partial y^{2}}+\rho_{1} v_{0} \frac{\partial T_{1}}{\partial y}-\mu_{1}\left[\left(\frac{\partial u_{1}}{\partial y}\right)^{2}+\left(\frac{\partial w_{1}}{\partial y}\right)^{2}\right]+ \\
& -\sigma_{l} u_{1}^{2} B_{0}^{2}-\sigma_{l} E_{0}^{2}-2 \sigma_{l} u_{1} B_{0} E_{0}=0 .
\end{aligned}
$$

Region II:

$$
\begin{aligned}
& \rho_{2} \frac{\partial u_{2}}{\partial t}-\mu_{2} \frac{\partial^{2} u_{2}}{\partial y^{2}}+\rho_{2} v_{0} \frac{\partial u_{2}}{\partial y}+\frac{\partial p}{\partial x}+\sigma_{2} u_{2} B_{0}^{2}+\sigma_{2} B_{0} E_{0}=-2 \rho_{2} \Omega w_{2}, \\
& \rho_{2} \frac{\partial w_{2}}{\partial t}-\mu_{2} \frac{\partial^{2} w_{2}}{\partial y^{2}}+\rho_{2} v_{0} \frac{\partial w_{2}}{\partial y}+\sigma_{2} w_{2} B_{0}^{2}=2 \rho_{2} \Omega u_{2},
\end{aligned}
$$




$$
\begin{aligned}
& \rho_{2} c_{p_{2}} \frac{\partial T_{2}}{\partial t}-K_{2} \frac{\partial^{2} T_{2}}{\partial y^{2}}+\rho_{2} \mathrm{v}_{0} \frac{\partial T_{2}}{\partial y}-\mu_{2}\left[\left(\frac{\partial u_{2}}{\partial y}\right)^{2}+\left(\frac{\partial w_{2}}{\partial y}\right)^{2}\right]+ \\
& -\sigma_{2} u_{2}^{2} B_{0}^{2}-\sigma_{2} E_{0}^{2}-2 \sigma_{2} u_{2} B_{0} E_{0}=0,
\end{aligned}
$$

in which, the subscripts 1 and 2 represent the quantities for Region-I and Region-II, respectively, where $u_{l}$, $u_{2}$ and $w_{1}, w_{2}$ are the $x$ - and $z$-components of fluid velocities, which are known as the primary and secondary velocity distributions in the two fluid regions respectively. $\Omega$ is the angular velocity, where $\bar{\Omega}=(0, \Omega, 0)$; $T_{1}, T_{2}$ are the fluid temperatures in the two regions, respectively and ' $t$ ' is the time. The boundary conditions on velocity are considered as the no-slip condition at the lower plate and an oscillatory type at the upper plate. The boundary conditions on temperature are assumed to be isothermal. It is also assumed the continuity of velocity, shear stress, temperature and heat flux at the interface between the two fluid layers (that is, at $y=0$ ).

The boundary and interface conditions on $u_{1}, w_{1}$ and $u_{2}, w_{2}$ for the fluids in the two regions are given by

$$
\begin{aligned}
& u_{l}\left(h_{l}\right) \quad \text { and } \quad w_{l}\left(h_{1}\right)=0 \quad \text { for } \quad t \leq 0, \\
& =\operatorname{Real}\left(\varepsilon e^{i \omega t}\right), \quad \text { for } \quad t>0 . \\
& u_{2}\left(-h_{2}\right)=0, \quad w_{2}\left(-h_{2}\right)=0, \\
& u_{l}(0)=u_{2}(0), \quad w_{1}(0)=w_{2}(0), \\
& \mu_{1} \frac{d u_{1}}{d y}=\mu_{2} \frac{d u_{2}}{d y} \quad \text { and } \quad \mu_{1} \frac{d w_{1}}{d y}=\mu_{2} \frac{d w_{2}}{d y} \quad \text { at } \quad y=0
\end{aligned}
$$

where $\varepsilon$ (amplitude) is a small constant quantity such that $\varepsilon<<1$ and $\omega$ is the frequency of oscillation at the plate and the perturbed fields initially are zero, as the system is at rest for $t \leq 0$.

Since the two plates are maintained at the same temperature, the boundary and interface conditions on temperature for both the fluids are given by

$$
\begin{aligned}
& T_{1}\left(h_{1}\right)=T_{w}, \\
& T_{2}\left(-h_{2}\right)=T_{w}, \\
& T_{1}(0)=T_{2}(0), \\
& K_{1} \frac{d T_{1}}{d y}=K_{2} \frac{d T_{2}}{d y} \quad \text { at } \quad y=0 .
\end{aligned}
$$

Further, the following non-dimensional variables are introduced 


$$
\begin{aligned}
& u_{1}^{\bullet}=\frac{u_{1}}{u_{p}}, \quad u_{2}^{\bullet}=\frac{u_{2}}{u_{p}}, \quad w_{1}^{\bullet}=\frac{w_{1}}{u_{p}}, \quad w_{2}^{\bullet}=\frac{w_{2}}{u_{p}}, \\
& y_{i}^{\bullet}=\left(\frac{y_{i}}{h_{i}}\right) \quad(i=1,2), t^{*}=\frac{v_{1} t}{h_{l}^{2}}, \quad \omega^{*}=\frac{\omega h_{1}^{2} \rho_{1}}{\mu_{l}},
\end{aligned}
$$

$u_{p}$ (characteristic velocity) $=\left(-\frac{\partial p}{\partial x}\right) \frac{h_{1}^{2}}{\mu_{l}}, M^{2}$ (Hartmann number) $=B_{0}^{2} h_{1}^{2}\left(\frac{\sigma_{l}}{\mu_{l}}\right), T^{3}$ (Taylor number or rotation parameter, this is the reciprocal of the Ekman number) $=h_{1}^{2} \frac{\Omega}{\mathrm{v}_{1}}, \lambda$ (porous parameter or suction number) $=h_{l} \rho_{1} v_{0} / \mu_{1}, \alpha$ (ratio of the viscosities) $=\frac{\mu_{1}}{\mu_{2}}, h$ (ratio of the heights) $=\frac{h_{2}}{h_{1}}, \sigma$ (ratio of the electrical conductivities) $=\frac{\sigma_{1}}{\sigma_{2}}, \beta$ (ratio of thermal conductivities) $=\frac{K_{1}}{K_{2}}, \theta_{i}=\frac{T_{i}-T_{w}}{u_{p}^{2} \mu_{1} / K_{i}}, R_{e}$ (electric load parameter) $=E_{0} / B_{0} u_{p}$.

With the use of transformations (2.15) and for simplicity neglecting the asterisks, the nondimensional form of Eqs (2.1) to (2.6) for the fluids in two regions are expressed as:

\section{Region-I:}

$$
\begin{aligned}
& \frac{d u_{1}}{d t}-\frac{d^{2} u_{1}}{d y^{2}}+\lambda \frac{d u_{1}}{d y}+M^{2}\left(R_{e}+u_{1}\right)-1=-2 T^{2} w_{1}, \\
& \frac{d w_{1}}{d t}-\frac{d^{2} w_{1}}{d y^{2}}+\lambda \frac{d w_{1}}{d y}+M^{2} w_{1}=2 T^{2} u_{1}, \\
& \frac{d \theta_{1}}{d t}-\frac{d^{2} \theta_{1}}{d y^{2}}+\lambda \frac{d \theta_{1}}{d y}-\left[\left(\frac{d u_{1}}{d y}\right)^{2}+\left(\frac{d w_{1}}{d y}\right)^{2}\right]-M^{2}\left[\left(R_{e}+u_{1}\right)^{2}+w_{1}^{2}\right]=0 .
\end{aligned}
$$

\section{Region-II:}

$$
\begin{aligned}
& \frac{d u_{2}}{d t}-\frac{d^{2} u_{2}}{d y^{2}}+\lambda \frac{d u_{2}}{d y}+M^{2} h^{2} \alpha \sigma\left(R_{e}+u_{2}\right)-\alpha h^{2}=-2 \rho \alpha h^{2} T^{2} w_{2}, \\
& \frac{d w_{2}}{d t}-\frac{d^{2} w_{2}}{d y^{2}}+\lambda \frac{d w_{2}}{d y}+M^{2} h^{2} \alpha \sigma w_{2}=2 \rho \alpha h^{2} T^{2} u_{2}, \\
& \frac{d \theta_{2}}{d t}-\frac{d^{2} \theta_{2}}{d y^{2}}+\lambda \frac{d \theta_{2}}{d y}-\frac{\beta}{\alpha}\left[\left(\frac{d u_{2}}{d y}\right)^{2}+\left(\frac{d w_{2}}{d y}\right)^{2}\right]-\beta \sigma h^{2} M^{2}\left[\left(R_{e}+u_{2}\right)^{2}+w_{2}^{2}\right]=0 .
\end{aligned}
$$


The non-dimensional forms of boundary conditions on the velocity, temperature and interface conditions become

$$
\begin{aligned}
& u_{l}(+1) \quad \text { and } \quad w_{1}(+1)=0 \quad \text { for } \quad t \leq 0 \text {, } \\
& =\operatorname{Re}\left(\varepsilon e^{i \omega t}\right), \quad \text { for } \quad t>0 . \\
& u_{2}(-1)=0, \quad w_{2}(-1)=0 \\
& u_{l}(0)=u_{2}(0), \quad w_{l}(0)=w_{2}(0) \\
& \frac{d u_{1}}{d y}=(1 / \alpha h) \frac{d u_{2}}{d y} \quad \text { and } \quad \frac{d w_{1}}{d y}=(1 / \alpha h) \frac{d w_{2}}{d y} \quad \text { at } \quad y=0 \\
& \theta_{1}(+1)=0 \\
& \theta_{2}(-1)=0 \\
& \theta_{1}(0)=\theta_{2}(0) \\
& \frac{d \theta_{1}}{d y}=(1 / \beta h) \frac{d \theta_{2}}{d y} \quad \text { at } \quad y=0
\end{aligned}
$$

The above mentioned conditions (2.23) represent the no-slip conditions at the lower plate and the conditions (2.22) are due to oscillation of the upper plate for any time $t$. Conditions (2.24) and (2.25) represent the continuity of velocities and shear stress, respectively, at the interface $y=0$. The conditions (2.26) and (2.27) represent the isothermal conditions, while the conditions (2.28) and (2.29) denote the continuity of temperatures and heat flux at the interface $y=0$, respectively.

\section{Solutions of the problem}

The effect of flow parameters on the fluid temperatures and the heat transferred between the fluids and the plates is considered. It is assumed that the thermal boundary conditions apply everywhere on the infinite channel plates and the thermal conduction in the flow direction is neglected. The governing momentum Eqs (2.16), (2.17) and (2.19), (2.20) along with the energy Eqs (2.18) and (2.21) are to be solved subject to the boundary and interface conditions (2.22) - (2.29) for the velocity and temperature distributions in both fluid regions. The resulting coupled partial differential equations are reduced to ordinary linear differential equations by assuming the following two term series

$$
\begin{aligned}
& u_{1}(y, t)=u_{01}(y)+\varepsilon \cos \omega t u_{11}(y), \\
& w_{1}(y, t)=w_{01}(y)+\varepsilon \cos \omega t w_{11}(y), \\
& u_{2}(y, t)=u_{02}(y)+\varepsilon \cos \omega t u_{12}(y), \\
& w_{2}(y, t)=w_{02}(y)+\varepsilon \cos \omega t w_{12}(y),
\end{aligned}
$$




$$
\begin{aligned}
& \theta_{1}(y, t)=\theta_{01}(y)+\varepsilon \cos \omega t \theta_{11}(y), \\
& \theta_{2}(y, t)=\theta_{02}(y)+\varepsilon \cos \omega t \theta_{12}(y),
\end{aligned}
$$

in which the terms $u_{01}(y), u_{02}(y), w_{01}(y), w_{02}(y)$ and $\theta_{01}(y), \theta_{02}(y)$ are velocity and temperature distributions in the basic steady state case in the two regions, while $u_{11}(y), u_{12}(y), w_{11}(y), w_{12}(y)$, and $\theta_{11}(y), \theta_{12}(y)$ are the corresponding time dependent components of the solutions, which are the factors of the real part $\left(\varepsilon e^{i \omega t}\right)$ to be determined with the help of Eqs (2.16) to (2.21).

By using the expressions given in Eqs (3.1) - (3.6) in Eqs (2.16) - (2.21), and separating the steady state and transient time varying components, the following differential equations in $u_{01}(y), u_{02}(y)$, $w_{01}(y), w_{02}(y)$ and $\theta_{01}(y), \theta_{02}(y)$; also, $u_{11}(y), u_{12}(y), w_{11}(y), w_{12}(y)$ and $\theta_{11}(y), \theta_{12}(y)$ are obtained in terms of the complex notations: $q_{01}=u_{01}+i w_{01}, q_{11}=u_{11}+i w_{11}, q_{02}=u_{02}+i w_{02}, q_{12}=u_{12}+i w_{12}$ for both the fluid regions as:

\section{Region-I}

\section{For the steady-state part}

$$
\begin{aligned}
& \frac{d^{2} q_{01}}{d y^{2}}-\lambda \frac{d q_{01}}{d y}-a_{1} q_{01}=a_{2}, \\
& \frac{d^{2} \theta_{01}}{d y^{2}}-\lambda \frac{d \theta_{01}}{d y}=-b_{22} e^{b_{5} y}-b_{23} e^{b_{6} y}-b_{24} e^{b_{7} y}+ \\
& -b_{25} e^{b_{8} y}-b_{26} e^{m_{1} y}-b_{27} e^{m_{2} y}-b_{28} e^{\bar{m}_{1} y}-b_{29} e^{\bar{m}_{2} y}-b_{2 i}
\end{aligned}
$$

\section{For the transient time dependent part}

$$
\begin{aligned}
& \frac{d^{2} q_{11}}{d y^{2}}-\lambda \frac{d q_{11}}{d y}-a_{3} \cdot q_{11}=0, \\
& \frac{d^{2} \theta_{11}}{d y^{2}}-b_{135} \frac{d \theta_{11}}{d y}+b_{136} \theta_{11}=-b_{137} e^{b_{115} y}-b_{138} e^{b_{116} y}-b_{139} e^{b_{117} y}+ \\
& -b_{140} e^{b_{118} y}-b_{141} e^{m_{5} y}-b_{142} e^{m_{6} y}-b_{143} e^{\bar{m}_{5} y}-b_{144} e^{\overline{m_{6} y}} .
\end{aligned}
$$

\section{Region - II}

\section{For the steady-state part}

$$
\begin{aligned}
& \frac{d^{2} q_{02}}{d y^{2}}-\lambda \frac{d q_{02}}{d y}-a_{4} q_{02}=a_{5}, \\
& \frac{d^{2} \theta_{02}}{d y^{2}}-\lambda \frac{d \theta_{02}}{d y}=-b_{53} e^{b_{36} y}-b_{54} e^{b_{37} y}-b_{55} e^{b_{38} y}-b_{56} e^{b_{39} y}+ \\
& -b_{57} e^{m_{3} y}-b_{58} e^{m_{4} y}-b_{59} e^{\bar{m}_{3} y}-b_{60} e^{\bar{m}_{4} y}-b_{52} .
\end{aligned}
$$




\section{For the transient time dependent part}

$$
\begin{aligned}
& \frac{d^{2} q_{12}}{d y^{2}}-\lambda \frac{d q_{12}}{d y}-a_{6} q_{12}=0, \\
& \frac{d^{2} \theta_{12}}{d y^{2}}-b_{152} \frac{d \theta_{12}}{d y}+b_{153} \theta_{12}=-b_{174} e^{b_{162} y}-b_{175} e^{b_{163} y}-b_{176} e^{b_{164} y}+ \\
& -b_{177} e^{b_{165} y}-b_{170} e^{m_{7} y}-b_{171} e^{m_{8} y}-b_{172} e^{\overline{m_{7} y}}-b_{173} e^{\overline{m_{8} y}} .
\end{aligned}
$$

The corresponding boundary and interface conditions on velocity and temperature become:

\section{For the steady-state part}

$$
\begin{aligned}
& q_{01}(+1)=0, \\
& q_{02}(-1)=0, \\
& q_{01}(0)=q_{02}(0), \\
& \frac{d q_{01}}{d y}=\frac{1}{\alpha h} \frac{d q_{02}}{d y} \quad \text { at } \quad y=0, \\
& \theta_{01}(+1)=0, \\
& \theta_{02}(-1)=0, \\
& \theta_{01}(0)=\theta_{02}(0), \\
& \frac{d \theta_{01}}{d y}=\frac{1}{\beta h} \frac{d \theta_{02}}{d y} \quad \text { at } \quad y=0 .
\end{aligned}
$$

\section{For the transient time dependent part}

$$
\begin{aligned}
& q_{11}(+1)=1, \\
& q_{12}(-1)=0, \\
& q_{11}(0)=q_{12}(0), \\
& \frac{d q_{11}}{d y}=\frac{1}{\alpha h} \frac{d q_{12}}{d y} \quad \text { at } \quad y=0 . \\
& \theta_{11}(1)=0, \\
& \theta_{12}(-1)=0, \\
& \theta_{11}(0)=\theta_{12}(0), \\
& \frac{d \theta_{11}}{d y}=\frac{1}{\beta h} \frac{d \theta_{12}}{d y} \quad \text { at } \quad y=0 .
\end{aligned}
$$


The differential equations given in Eqs (3.7) - (3.14) along with the boundary and interface conditions from Eqs (3.15) to (3.22) represent a system of ordinary linear differential equations and conditions. These equations are solved in a closed form for both the steady state and transient time dependent components separately in two parts. Hence, by making use of the solutions of velocity distributions, it is obtained the solutions for temperature distributions of the unsteady flow problem in both fluid regions as:

\section{$\underline{\text { Region-I }}$}

$$
\begin{aligned}
& \theta_{1}(y, t)=\theta_{01}(y)+\operatorname{Real}\left(\varepsilon e^{i \omega t}\right) \theta_{11}(y) \\
& =d_{1}+d_{2} e^{\lambda y}+b_{61} e^{b_{5} y}+b_{62} e^{b_{6} y}+b_{63} e^{b_{7} y}+b_{64} e^{b_{8} y}+b_{65} e^{m_{1} y}+b_{66} e^{m_{2} y}+ \\
& +b_{67} e^{m_{1} y}+b_{68} e^{m_{2} y}+b_{69} y+\operatorname{Re}\left(\varepsilon \mathrm{e}^{\mathrm{i} \omega \mathrm{t}}\right)\left(d_{5} e^{m_{9} y}+d_{6} e^{m_{10} y}+b_{178} e^{b_{115} y}+b_{179} e^{b_{116} y}+\right. \\
& \left.+b_{180} e^{b_{117} y}+b_{181} e^{b_{118} y}+b_{182} e^{m_{5} y}+b_{183} e^{m_{6} y}+b_{184} e^{\bar{m}_{5} y}+b_{185} e^{\bar{m}_{6} y}\right)
\end{aligned}
$$

\section{$\underline{\text { Region-II }}$}

$$
\begin{aligned}
& \theta_{2}(y, t)=\theta_{02}(y)+\operatorname{Real}\left(\varepsilon e^{i \omega t}\right) \theta_{12}(y) \\
& =d_{3}+d_{4} e^{\lambda y}+b_{78} e^{b_{36} y}+b_{79} e^{b_{37} y}+b_{80} e^{b_{38} y}+b_{81} e^{b_{39} y}+b_{82} e^{m_{3} y}+ \\
& +b_{83} e^{m_{4} y}+b_{84} e^{\bar{m}_{3} y}+b_{85} e^{\bar{m}_{4 y} y}+b_{86} y+ \\
& +\operatorname{Re}\left(\varepsilon \mathrm{e}^{\mathrm{i} \omega \mathrm{t}}\right)\left(d_{7} e^{m_{11} y}+d_{8} e^{m_{12} y}+b_{194} e^{b_{162} y}+b_{195} e^{b_{163} y}+b_{196} e^{b_{164} y}+\right. \\
& \left.+b_{197} e^{b_{165} y}+b_{198} e^{m_{7} y}+b_{199} e^{m_{8} y}+b_{200} e^{\overline{m_{7} y}}+b_{201} e^{\overline{m_{8} y}}\right)
\end{aligned}
$$

The solutions of the non-periodic terms represent the steady-state fluid flow solutions for both regions. The steady-state temperature profiles are shown in Figs 2 to 8 as dash-dot lines. The solution of the periodic terms gives the transient velocity and hence the temperature distribution for both fluid regions of the channel is obtained. The solutions given in Eqs (3.31) to (3.32) for temperature distribution of the unsteady problem are evaluated numerically for different non-dimensional governing flow parameters involved in the study. Also, these results are plotted and shown as solid lines in Figs 2 to 8 . The value for $\varepsilon$ is fixed at 0.5 , $\mathrm{R}_{\mathrm{e}}=-1$ and $P_{r}=1$ for all graphs. The constants appearing in the above solutions are given in the Appendix.

\section{Results and discussion}

The solutions for the temperature distributions in the two fluid regions, namely $\theta_{1}, \theta_{2}$, are given analytically for small $\varepsilon$, the coefficient of exponent of periodic frequency parameter. The corresponding profiles are plotted after obtaining the computational values for different sets of values of the governing parameters involved, such as the Hartmann number ' $\mathrm{M}$ ', Taylor number (rotation parameter) ' $\mathrm{T}$ ', suction number (porous parameter) $\lambda$, ratios of the: viscosities $\alpha$, heights ' $h$ ', electrical conductivities $\sigma$ and the thermal conductivities $\beta$. The results are depicted graphically in Figs $2-8$ for the temperature distributions in Region-I and Region-II to elucidate the important features of the magnetohydrodynamic thermal state of the flow. The solid lines show the profiles for an unsteady flow and the dash-dot lines for the steady motions respectively. We note that when the motion is in a steady state and $T=0, \lambda=0$ (i.e., without rigid rotation and non-porous boundaries), these results coincide with those of Malashetty and Leela [12]. Also, when $T=0$, (i.e., for porous plates and without rigid rotation) the analysis is in agreement with the solutions of Raju and Sreedhar [47].

Figure 2 provides the graphical illustration for the effect of different values of the Hartmann number $M$ on the dimensionless temperature distributions $\theta_{1}, \theta_{2}$ in the two fluid regions (Region-I and Region-II) for fixed 
values of the Taylor number, porous parameter, and ratios of the viscosities, heights, electrical and thermal conductivities. It is found that an increase in ' $\mathrm{M}$ ' enhances the temperature distribution in the two regions and the maximum temperature in the channel tends to move above the channel centre line towards Region-I.

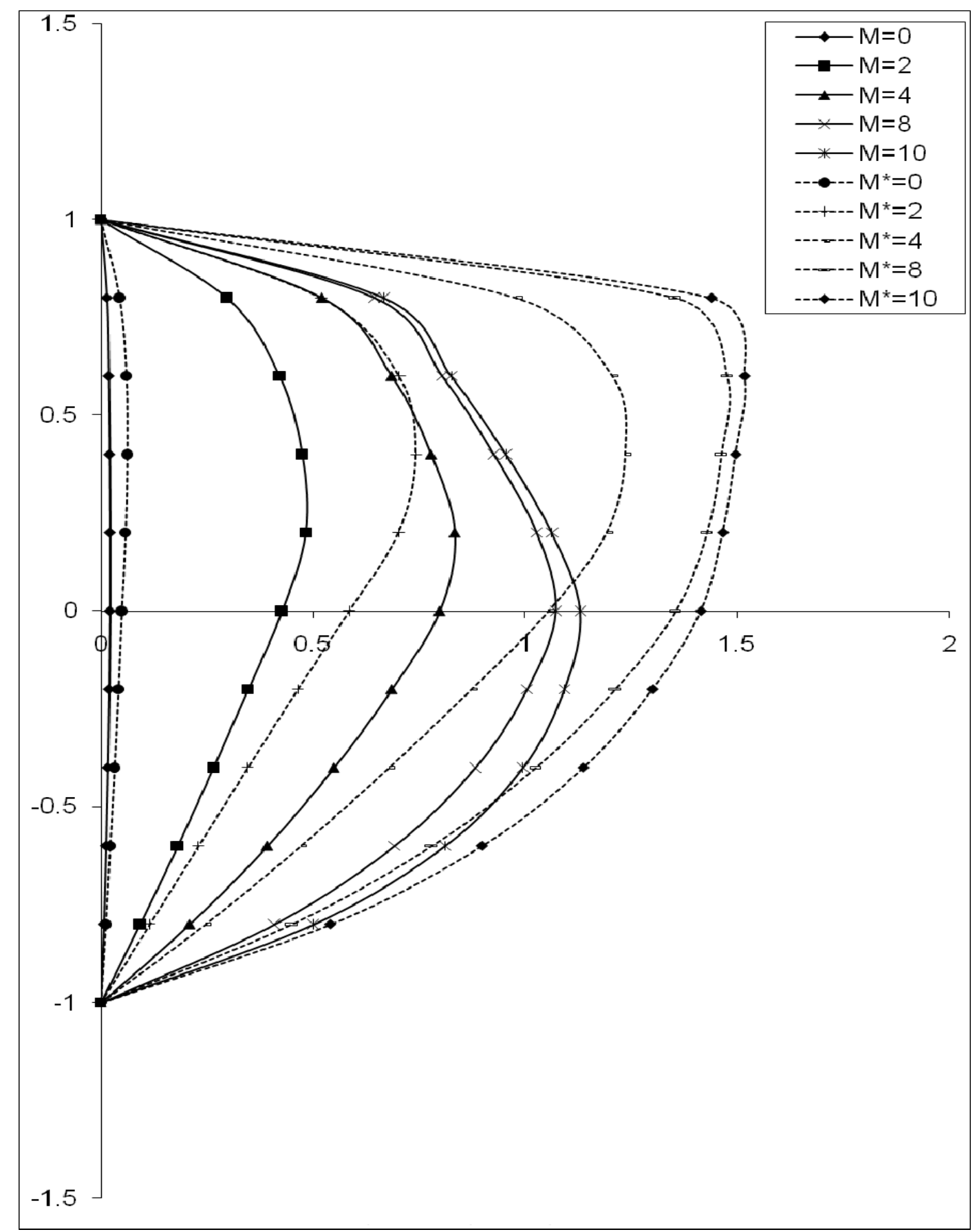

Fig.2. Temperature profiles $\theta 1, \theta 2$ (unsteady flow), $\theta 1^{*}, \theta 2^{*}$ (steady flow), for different $\mathrm{M}$ and $\lambda=1$, $\beta=1, \rho=1.5, k=0.8, \alpha=0.333, \sigma=0.1, \operatorname{Re}=-1, h=0.75, \varepsilon=0.5, \omega=1, t=\pi / \omega$.

The effect of the Taylor number (rotation parameter)' T" on temperature distribution in the two-fluid regions is exhibited in Fig.3. It is seen that an increase in ' $\mathrm{T}$ ' increases the temperature distribution in both 
the regions. Also when ' $\mathrm{T}$ ' increases, the maximum temperature in the channel tends to move above the channel centre line towards region-I. This may be due to the fact that, a strong Coriolis force is generated from a higher angular velocity of rotation which may transform the magnetic energy of the magnetic field into kinetic energy for pushing up of the fluid particles to acquire higher velocity components in turn resulting higher temperature distributions in the two fluid regions.

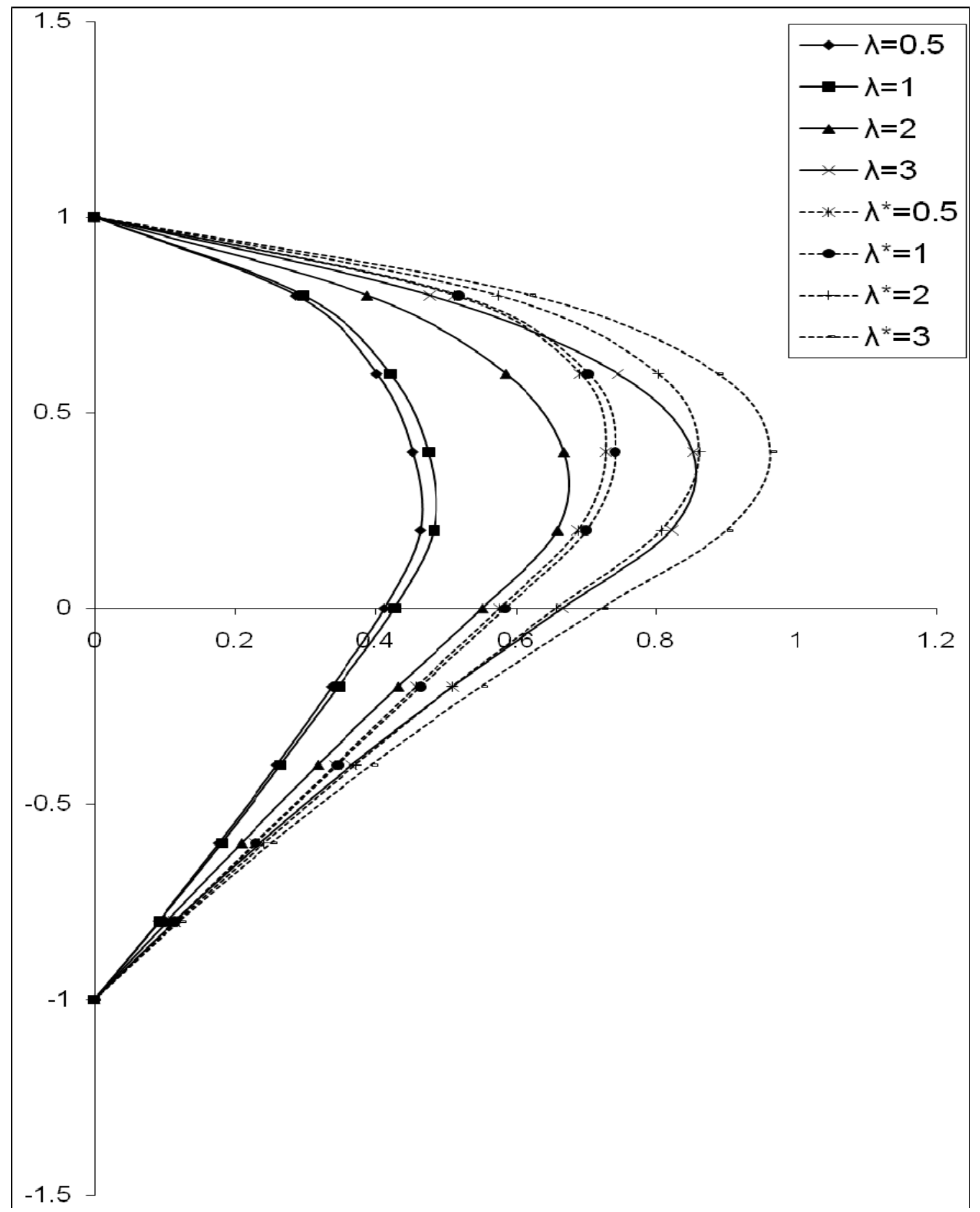

Fig.3. Temperature profiles $\theta 1, \theta 2$ (unsteady flow), $\theta 1^{*}, \theta 2^{*}$ (steady flow), for different $\lambda$ and $\mathrm{M}=2$, $\beta=1, \rho=1.5, k=0.8, \alpha=0.333, \sigma=0.1, \operatorname{Re}=-1, h=0.75, \varepsilon=0.5, \omega=1, t=\pi / \omega$. 
Figure 4 demonstrates the effect of varying the porous parameter (suction number) $\lambda$ on the temperature distribution in the two-fluid regions. It is observed that an increase in $\lambda$ diminishes the temperature distribution in both fluid regions. And the maximum temperature in the channel tends to move above the channel centre line towards region-I as $\lambda$ increases. This is due to the fact that, when the suction number increases, the velocity components become thin and hence a decrease in temperature distribution.

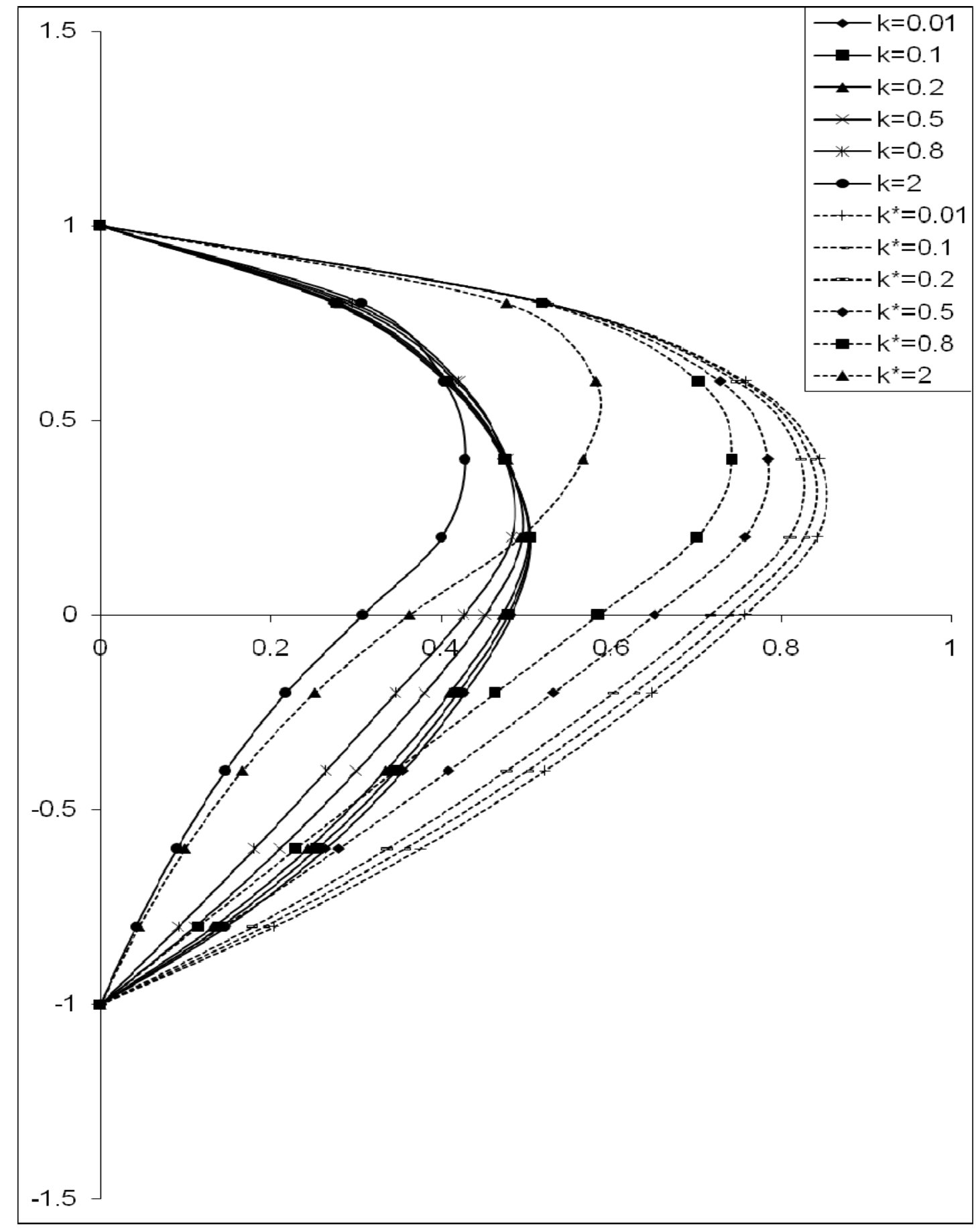

Fig.4. Temperature profiles $\theta 1, \theta 2$ (unsteady flow), $\theta 1^{*}, \theta 2^{*}$ (steady flow), for different $k$ and $\mathrm{M}=2, \beta=1$, $\lambda=1, \rho=1.5, \alpha=0.333, \sigma=0.1, \operatorname{Re}=-1, h=0.75, \varepsilon=0.5, \omega=1, t=\pi / \omega$. 
Figure 5 exhibits the effect of the different values of the electrical conductivity ratio $\sigma$ on temperature distributions. It is observed that an increase in $\sigma$ increases the temperature distribution in the two regions. The maximum temperature in the channel tends to move below the channel centre line towards Region-II as $\sigma$ increases.

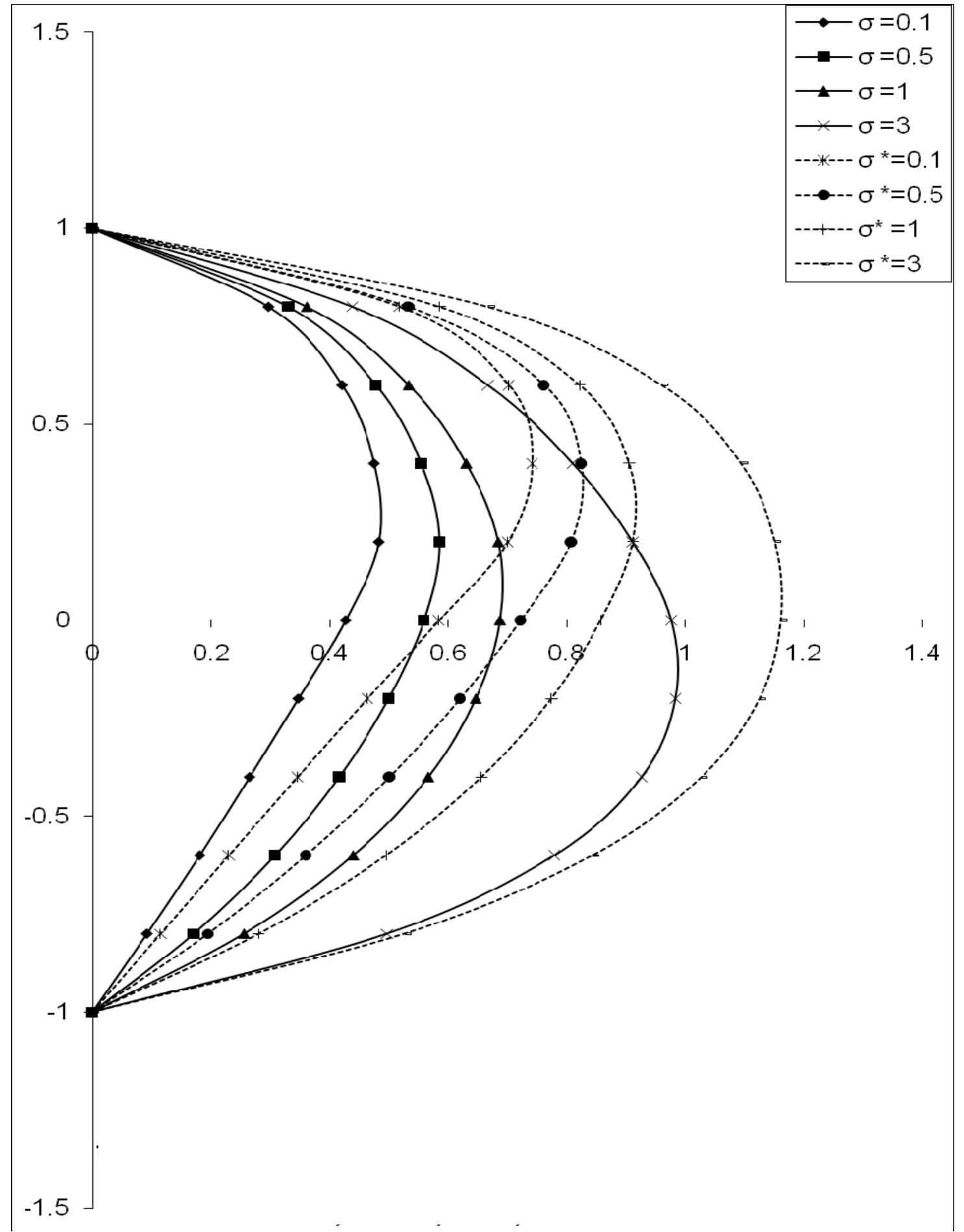

Fig.5. Temperature profiles $\theta 1, \theta 2$ (unsteady flow), $\theta 1^{*}, \theta 2^{*}$ (steady flow), for different $\sigma$ and $\mathrm{M}=2$, $\beta=1, \lambda=1, \rho=1.5, k=0.8, \alpha=0.333, \operatorname{Re}=-1, h=0.75, \varepsilon=0.5, \omega=1, t=\pi / \omega$. 
Figure 6 shows the effect of viscosity ratio $\alpha$ on the temperature distribution. It is observed that an increase in $\alpha$ diminishes the temperature distribution in the two regions. And the maximum temperature distribution in the channel tends to move above the channel centre line towards Region-I. But for small values of $\alpha$, more heat is added to the fluid due to viscous heating and hence the temperature in the two regions increase with decreasing values of $\alpha$.

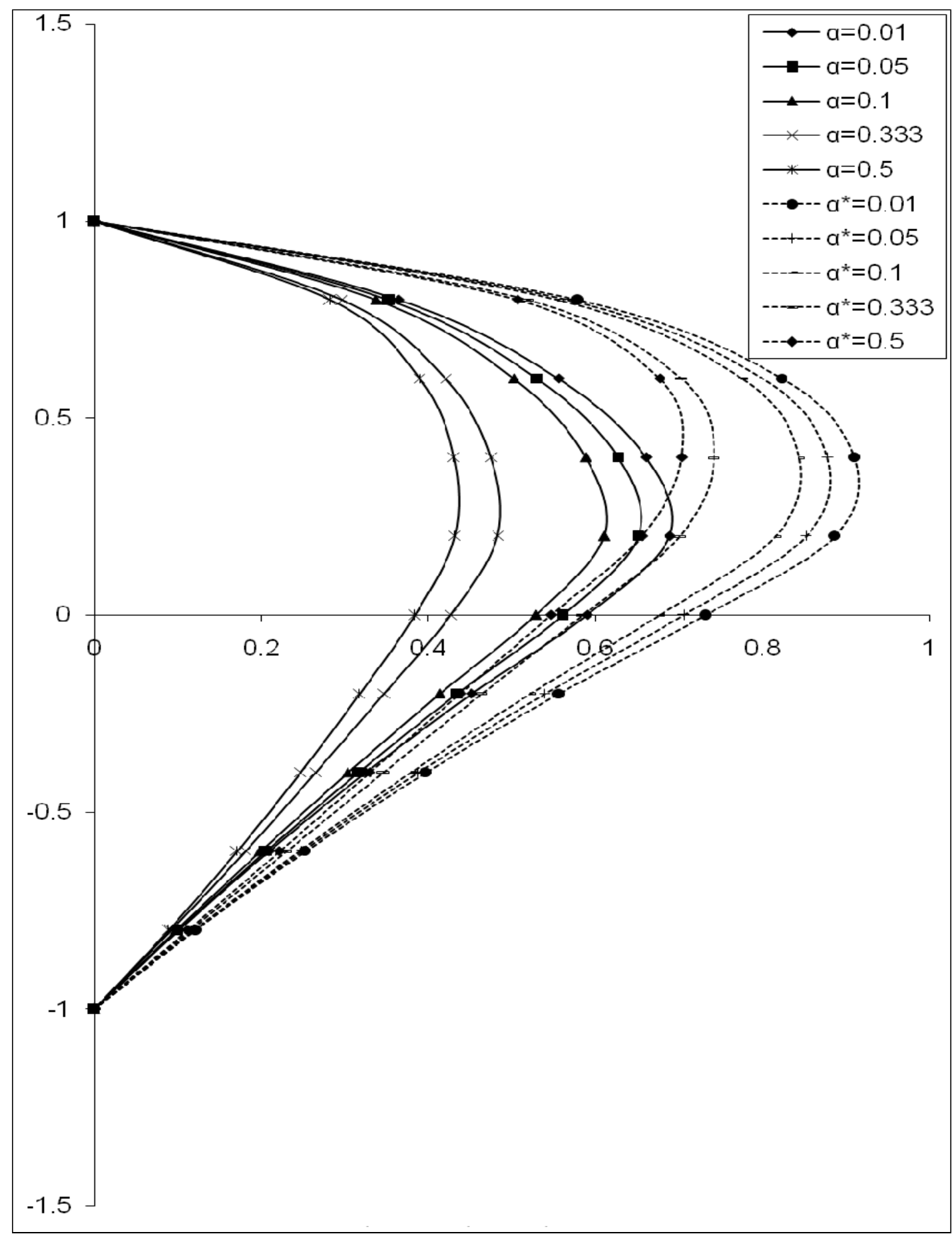

Fig.6. Temperature profiles $\theta 1, \theta 2$ (unsteady flow), $\theta 1^{*}, \theta 2^{*}$ (steady flow), for different $\alpha$ and $\mathrm{M}=2$, $\beta=1, \lambda=1, k=0.8, \rho=1.5, \sigma=0.1, \operatorname{Re}=-1, h=0.75, \varepsilon=0.5, \omega=1, t=\pi / \omega$. 
The effect of varying the height ratio ' $h$ ' on temperature distribution is shown in the Fig.7. It is observed that an increase in ' $h$ ' increases the temperature distribution in the two regions. Also, the maximum temperature in the channel tends to move above the channel centre line towards Region-I as ' $h$ ' increases.

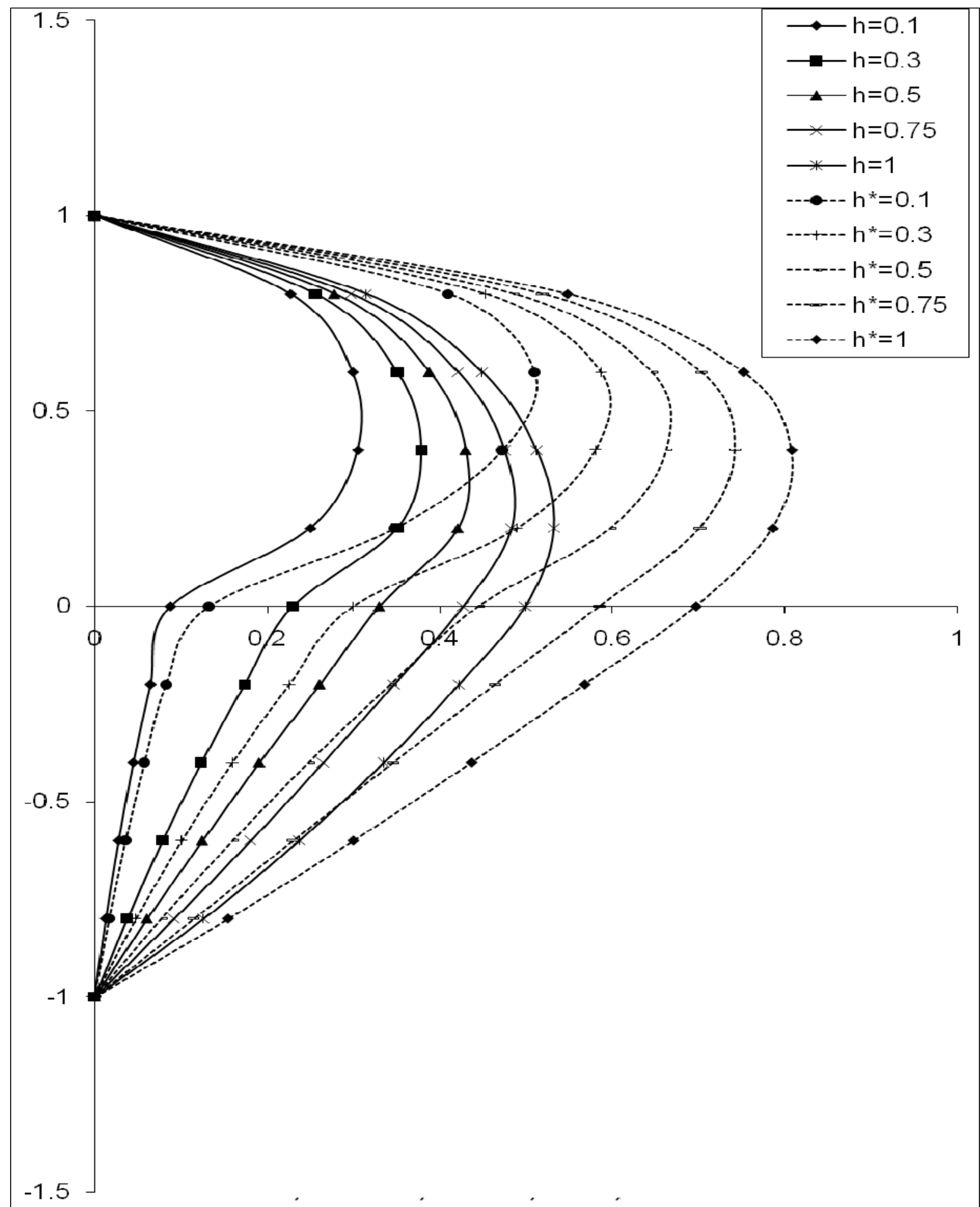

Fig.7. Temperature profiles $\theta 1, \theta 2$ (unsteady flow), $\theta 1^{*}, \theta 2^{*}$ (steady flow), for different $h$ and $\mathrm{M}=2, \beta=1$, $\lambda=1, \rho=1.5, k=0.8, \alpha=0.333, \operatorname{Re}=-1, \sigma=0.1, \varepsilon=0.5, \omega=1, t=\pi / \omega$. 
Figure 8 exhibits the effect of varying the thermal conductivity ratio $\beta$ on the temperature distribution. It is observed that an increasing $\beta$ increases the temperature distribution in the two fluid regions. Also, the maximum temperature in the channel tends to move slightly above the channel centre line towards Region-I as $\beta$ increases.

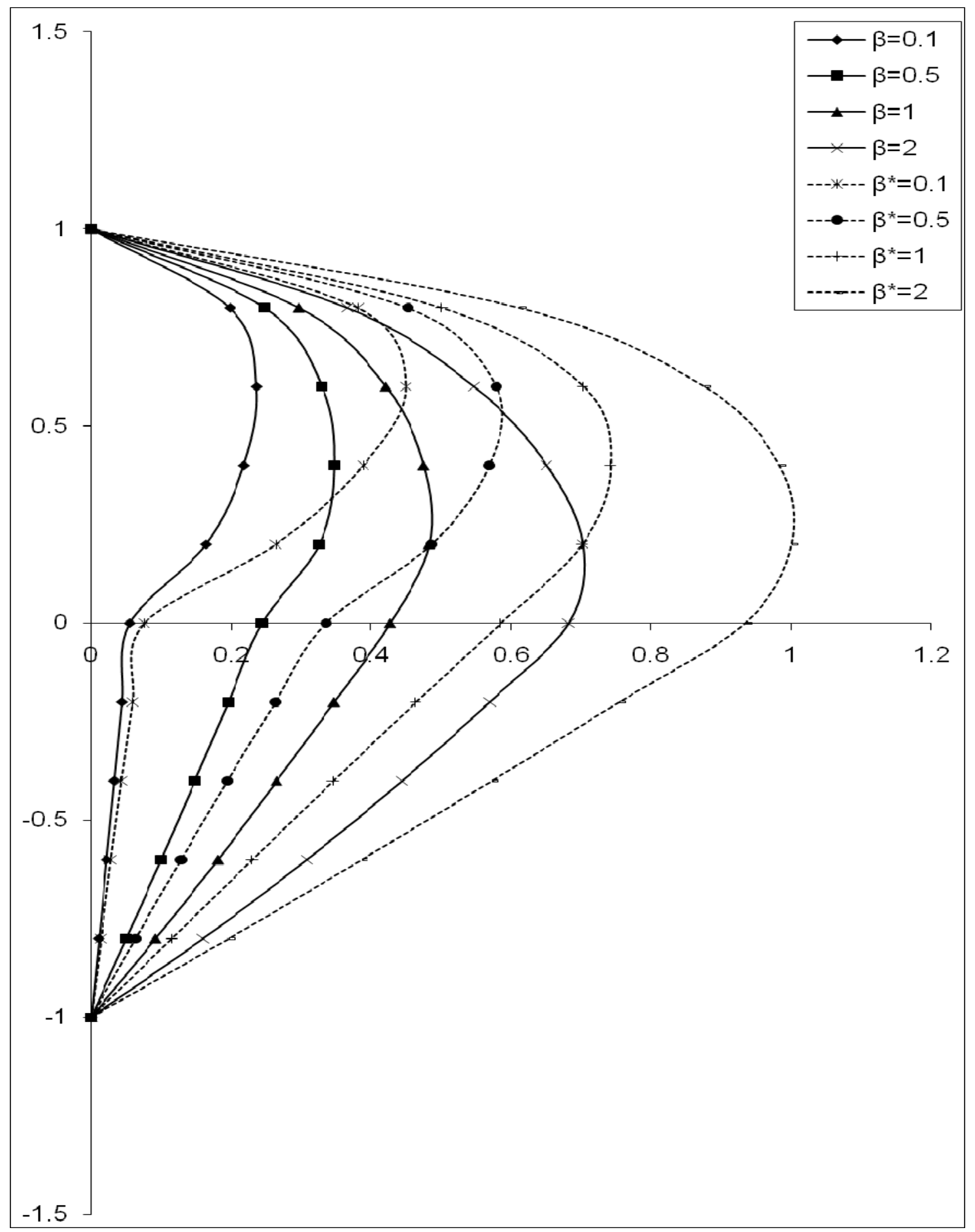

Fig.8. Temperature profiles $\theta 1, \theta 2$ (unsteady flow), $\theta 1^{*}, \theta 2^{*}$ (steady flow), for different $\beta$ and $M=2$, $h=0.75, \lambda=1, \rho=1.5, k=0.8, \alpha=0.333, \operatorname{Re}=-1, \sigma=0.1, \varepsilon=0.5, \omega=1, t=\pi / \omega$. 


\section{Conclusion}

The problem of an unsteady magnetohydrodynamic (MHD) flow of two-layered fluids through horizontal channel bounded by two parallel porous plates in the presence of an applied magnetic and electric fields in a rotating frame of reference is analyzed theoretically. The transpiration velocity and temperature are assumed to vary periodically with time about a non-zero velocity and temperature. The analytical solutions of the governing equations are evaluated numerically to plot their graphs for the temperature distributions in both the fluid regions. The combined effect of the magnetic field and Coriolis force in porous channels on the temperature profiles in both the fluid regions is also discussed. Comparisons with previously published theoretical works are performed and an excellent agreement is obtained with the earlier studies for the fluid flows over porous boundaries in the absence of rigid rotation. It is found that an increase in the Hartmann number ' $M$ ' rises the temperature in the two regions for fixed values of the remaining governing parameters involved. Also, as' $\mathrm{M}$ ' increases the maximum temperature in the channel tends to move above the channel centre line towards Region-I. It is observed that as the Coriolis forces become stronger, that is as the Taylor number $\mathrm{T}$ increases, the temperature increases in both the fluid regions. The maximum temperature in the channel tends to move above the channel centre line towards Region- $\mathrm{I}$ as ' $\mathrm{T}$ ' increases. This shows that a strong Coriolis force can change the mode of action of the magnetic field in the presence of plate/wall porosity. It is also seen that, an increase in porous parameter diminishes the temperature distribution in both the regions. And it is noticed that the temperatures in the two regions can be enhanced with the suitable values of the ratios of heights, electrical and thermal conductivities. Further, a comparison shows that the temperature profiles in an unsteady state flow problem are pronounced less when compared to the steady state two-layered fluid motion.

\section{Nomenclature}

$$
\begin{aligned}
& a_{1}, a_{2,}, ., c_{1}, c_{2}, \ldots \quad-\text { functions / real constants represented in the equations and solutions } \\
& B_{0}-\text { applied uniform transverse magnetic field } \\
& E_{0} \text { - constant electric field in the z-direction } \\
& h \text { - ratio of the heights of the two regions } \\
& h_{1} \text { - height of the channel in the upper region } \\
& h_{2} \text { - height of the channel in the lower region } \\
& K_{1}, K_{2} \text { - thermal conductivities of the two fluids } \\
& \mathrm{M} \text { - Hartmann number } \\
& P \text { - pressure } \\
& \text { Pr - Prandtl number } \\
& R_{e} \text { - electric load parameter } \\
& \mathrm{T} \text { - Taylor number (rotation parameter) } \\
& T_{1}, T_{2} \text { - temperatures of the fluids in the two regions respectively } \\
& T_{w_{1}}, T_{w_{2}} \text { - constant temperatures at both the walls } \\
& t \text { - time } \\
& u_{1}, u_{2}-x \text { - component of velocity distributions in the two fluid regions, which are known as the primary } \\
& \text { velocity distributions } \\
& u_{01}(y), u_{02}(y) \text { - primary velocity distributions in the basic steady state case in two regions } \\
& u_{11}(y), u_{12}(y) \text { - time dependent primary velocity components } \\
& u_{p} \quad-=\left(-\frac{\partial p}{\partial x}\right) \frac{h_{1}^{2}}{\mu_{1}}, \text { the characteristic velocity } \\
& v_{0}-\text { constant suction velocity }
\end{aligned}
$$


$w_{1}, w_{2}-z^{-}$component of velocity distributions in the two fluid regions, called the secondary velocity distributions

$w_{01}(y), w_{02}(y)$ - secondary velocity distributions in the basic steady state case in two regions

$w_{11}(y), w_{12}(y)$ - time dependent secondary velocity components

$(x, y, z)$ - space co-ordinates

$\alpha$ - ratio of viscosities

$\beta-$ ratio of thermal conductivities

$\varepsilon \quad$ amplitude (a small constant quantity such that $\varepsilon<<1$ )

$\theta_{1}, \theta_{2}-$ non-dimensional forms of temperature distributions of the two fluids

$\theta_{01}(y), \theta_{02}(y)$ - temperature distributions in the basic steady state case in the two regions

$\theta_{11}(y), \theta_{12}(y)$ - time dependent components of the temperatures in the two regions

$\lambda$ - porous parameter (suction number)

$\mu_{1}, \mu_{2}-$ viscosities of the two fluids

$\rho_{1}, \rho_{2}-$ densities of the two fluids

$\sigma$ - ratio of electrical conductivities

$\sigma_{1}, \sigma_{2}-$ electrical conductivities of the two fluids

$\Omega$ - angular velocity, where $\bar{\Omega}=(0, \Omega, 0)$

$\omega-$ frequency of oscillation

\section{Subscripts}

1,2 - refers to the quantities in the upper and lower fluid regions respectively

\section{Appendix}

$$
\begin{aligned}
& a_{1}=M^{2}+2 T^{2} i, \quad a_{2}=M^{2} R_{e}-1, \quad a_{3}=a_{1}-\omega \tan \omega, \quad a_{4}=M^{2} h^{2} \alpha \sigma+i 2 \rho \alpha h^{2} T^{2}, \\
& a_{5}=M^{2} h^{2} \alpha \sigma R_{e}-\alpha h^{2}, \quad a_{6}=a_{4}-\omega \tan \omega t, \\
& m_{1}=\frac{\lambda+\sqrt{\lambda^{2}+4 a_{1}}}{2}, \quad m_{2}=\frac{\lambda-\sqrt{\lambda^{2}+4 a_{1}}}{2}, \quad m_{3}=\frac{\lambda+\sqrt{\lambda^{2}+4 a_{4}}}{2}, \quad m_{4}=\frac{\lambda-\sqrt{\lambda^{2}+4 a_{4}}}{2}, \\
& a_{7}=e^{m_{1}-m_{2}}, \quad a_{8}=\frac{a_{2}}{a_{1}} e^{-m_{2}}, \quad a_{9}=e^{-m_{3}+m_{4}}, \quad a_{10}=\frac{a_{5}}{a_{4}} e^{m_{4}}, \quad a_{11}=\frac{-a_{5}}{a_{4}}+\frac{a_{2}}{a_{1}}, \\
& a_{12}=1-a_{7}, \quad a_{13}=-1+a_{9}, \quad a_{14}=a_{11}+a_{10}-a_{8}, \quad a_{15}=m_{1}-m_{2} a_{7}, a_{16}=\frac{1}{\alpha h}\left(m_{3}-m_{4} a_{9}\right), \\
& a_{17}=\frac{m_{4} a_{10}}{\alpha h}-m_{2} a_{8}, \quad a_{18}=\frac{a_{14} a_{15}-a_{12} a_{17}}{a_{12} a_{15}+a_{12} a_{16}}, a_{19}=\frac{a_{17}+a_{16} a_{18}}{a_{15}}, c_{2}=-c_{1} a_{7}+a_{8}, c_{1}=a_{19}, \\
& c_{4}=-c_{3} a_{9}+a_{10}, \quad c_{3}=a_{18}, \quad m_{5}=\frac{\lambda+\sqrt{\lambda^{2}+4 a_{3}}}{2}, \quad m_{6}=\frac{\lambda-\sqrt{\lambda^{2}+4 a_{3}}}{2},
\end{aligned}
$$




$$
\begin{aligned}
& m_{7}=\frac{\lambda+\sqrt{\lambda^{2}+4 a_{6}}}{2}, \quad m_{8}=\frac{\lambda-\sqrt{\lambda^{2}+4 a_{6}}}{2}, \quad a_{20}=e^{m_{5}-m_{6}}, \quad a_{21}=e^{-m_{6}}, \quad a_{22}=e^{-m_{8}+m_{7}}, \\
& a_{23}=1-a_{20}, \quad a_{24}=1-a_{22}, \quad a_{25}=m_{5}-m_{6} a_{20}, \quad a_{26}=\frac{1}{\alpha h}\left(-m_{7} a_{22}+m_{8}\right), \quad a_{27}=m_{6} a_{21}, \\
& a_{28}=\frac{a_{23} a_{27}-a_{21} a_{25}}{a_{23} a_{26}-a_{24} a_{25}}, \quad a_{29}=\frac{-a_{27}+a_{28} a_{26}}{a_{25}}, \quad c_{5}=a_{29}, \quad c_{6}=-c_{5} a_{20}+a_{21}, \quad c_{7}=-c_{8} a_{22}, \\
& c_{8}=a_{28}, \quad q_{11}(y)=c_{5} e^{m_{5} y}+c_{6} e^{m_{6} y}, \quad q_{12}(y)=c_{7} e^{m_{7} y}+c_{8} e^{m_{8} y}, \quad b_{1}=m_{1} c_{1} \overline{m_{1}} \overline{c_{1}}, \\
& b_{2}=m_{1} c_{1} \overline{m_{2}} \bar{c}_{2}, \quad b_{3}=m_{2} c_{2} \overline{m_{1}} \overline{c_{1}}, \quad b_{4}=m_{2} c_{2} \overline{m_{2}} \overline{c_{2}}, \quad b_{5}=m_{1}+\overline{m_{1}}, \quad b_{6}=m_{1}+\overline{m_{2}} \text {, } \\
& b_{7}=m_{2}+\overline{m_{1}}, \quad b_{8}=m_{2}+\overline{m_{2}}, \quad b_{9}=M^{2} c_{1} \overline{c_{1}}, \quad b_{10}=M^{2} c_{1} \overline{c_{2}}, \quad b_{11}=M^{2} c_{2} \overline{c_{1}}, \\
& b_{12}=M^{2} c_{2} \overline{c_{2}}, \quad b_{13}=M^{2} c_{1} \frac{\overline{a_{2}}}{\overline{a_{1}}}, \quad b_{14}=M^{2} c_{2} \frac{\overline{a_{2}}}{\overline{a_{1}}}, \quad b_{15}=M^{2} c_{1} \frac{a_{2}}{a_{1}}, \quad b_{16}=M^{2} \overline{c_{2}} \frac{a_{2}}{a_{1}}, \\
& b_{17}=M^{2} c_{1} R_{e}, \quad b_{18}=M^{2} c_{2} R_{e}, \quad b_{19}=M^{2} \overline{c_{1}} R_{e}, \quad b_{20}=M^{2} \overline{c_{2}} R_{e}, \\
& b_{21}=M^{2} \frac{a_{2}}{a_{1}} \frac{\overline{a_{2}}}{\overline{a_{1}}}-M^{2} R_{e} \frac{a_{2}}{a_{1}}-M^{2} R_{e}{ }^{2} \frac{\overline{a_{2}}}{\overline{a_{1}}}+M^{2} R_{e}^{2}, \quad b_{22}=b_{1}+b_{9}, \quad b_{23}=b_{2}+b_{10}, \\
& b_{24}=b_{3}+b_{11}, \quad b_{25}=b_{4}+b_{12}, \quad b_{26}=-b_{13}+b_{17}, \quad b_{27}=-b_{14}+b_{18}, \quad b_{28}=-b_{15}+b_{19}, \\
& b_{29}=-b_{16}+b_{20}, \quad b_{30}=\frac{\beta}{\alpha}, \quad b_{31}=M^{2} h^{2} \sigma \beta, \quad b_{32}=b_{30} c_{3} m_{3} \overline{c_{3}} \overline{m_{3}}, \quad b_{33}=b_{30} c_{3} m_{3} \overline{c_{4}} \overline{m_{4}}, \\
& b_{34}=b_{30} c_{4} m_{4} \overline{c_{3}} \overline{m_{3}}, \quad b_{35}=b_{30} c_{4} m_{4} \overline{c_{4}} \overline{m_{4}}, \quad b_{36}=m_{3}+\overline{m_{3}}, \quad b_{37}=m_{3}+\overline{m_{4}}, \\
& b_{38}=m_{4}+\overline{m_{3}}, \quad b_{39}=m_{4}+\overline{m_{4}}, \quad b_{40}=b_{31} c_{3} \overline{c_{3}}, \quad b_{41}=b_{31} c_{3} \overline{c_{4}}, \quad b_{42}=b_{31} c_{4} \overline{c_{3}}, \\
& b_{43}=b_{31} c_{4} \overline{c_{4}}, \quad b_{44}=b_{31} c_{3} \frac{\overline{a_{5}}}{\overline{a_{4}}}, \quad b_{45}=b_{31} c_{4} \frac{\overline{a_{5}}}{\overline{a_{4}}}, \quad b_{46}=b_{31} \overline{c_{3}} \frac{a_{5}}{a_{4}}, \quad b_{47}=b_{31} \overline{c_{4}} \frac{a_{5}}{a_{4}}, \\
& b_{48}=b_{31} c_{3} R_{e}, \quad b_{49}=b_{31} c_{4} R_{e}, \quad b_{50}=b_{31} \overline{c_{3}} R_{e}, \quad b_{51}=b_{31} \overline{c_{4}} R_{e} \text {, } \\
& b_{52}=b_{31} R_{e}^{2}+b_{31} \frac{a_{5}}{a_{4}} \frac{\overline{a_{5}}}{\overline{a_{4}}}-b_{31} R_{e} \frac{a_{5}}{a_{4}}-b_{31} R_{e} \frac{\overline{a_{5}}}{\overline{a_{4}}}, \quad b_{53}=b_{32}+b_{40}, \quad b_{54}=b_{33}+b_{41}, \\
& b_{55}=b_{34}+b_{42}, \quad b_{56}=b_{35}+b_{43}, \quad b_{57}=-b_{44}+b_{48}, \quad b_{58}=-b_{45}+b_{49}, \quad b_{59}=-b_{46}+b_{50} \text {, }
\end{aligned}
$$




$$
\begin{aligned}
& b_{60}=-b_{47}+b_{51}, \quad b_{61}=\frac{-b_{22}}{b_{5}{ }^{2}-\lambda b_{5}}, \quad b_{62}=\frac{-b_{23}}{b_{6}{ }^{2}-\lambda b_{6}}, \quad b_{63}=\frac{-b_{24}}{b_{7}{ }^{2}-\lambda b_{7}}, \quad b_{64}=\frac{-b_{25}}{b_{8}{ }^{2}-\lambda b_{8}}, \\
& b_{65}=\frac{-b_{26}}{m_{1}{ }^{2}-\lambda m_{1}}, \quad b_{66}=\frac{-b_{27}}{m_{2}{ }^{2}-\lambda m_{2}}, \quad b_{67}=\frac{-b_{28}}{{\overline{m_{1}}}^{2}-\lambda \overline{m_{1}}}, \quad b_{68}=\frac{-b_{29}}{{\overline{m_{2}}}^{2}-\lambda \overline{m_{2}}}, \quad b_{69}=\frac{b_{21}}{\lambda} \text {, } \\
& b_{70}=b_{61} b_{5}, \quad b_{71}=b_{62} b_{6}, \quad b_{72}=b_{63} b_{7}, \quad b_{73}=b_{64} b_{8}, \quad b_{74}=b_{65} m_{1}, \quad b_{75}=b_{66} m_{2}, \\
& b_{76}=b_{67} \overline{m_{1}}, \quad b_{77}=b_{68} \overline{m_{2}}, \quad b_{78}=\frac{-b_{53}}{b_{36}{ }^{2}-\lambda b_{36}}, \quad b_{79}=\frac{-b_{54}}{b_{37}{ }^{2}-\lambda b_{37}}, \quad b_{80}=\frac{-b_{55}}{b_{38}{ }^{2}-\lambda b_{38}}, \\
& b_{81}=\frac{-b_{56}}{b_{39}{ }^{2}-\lambda b_{39}}, \quad b_{82}=\frac{-b_{57}}{m_{3}^{2}-\lambda m_{3}}, \quad b_{83}=\frac{-b_{58}}{m_{4}{ }^{2}-\lambda m_{4}}, \quad b_{84}=\frac{-b_{59}}{{\overline{m_{3}}}^{2}-\lambda \overline{m_{3}}}, \\
& b_{85}=\frac{-b_{60}}{{\overline{m_{4}}}^{2}-\lambda \overline{m_{4}}}, \quad b_{86}=\frac{b_{52}}{\lambda}, \quad b_{87}=b_{78} b_{36}, \quad b_{88}=b_{79} b_{37}, \quad b_{88}=b_{79} b_{37}, \quad b_{89}=b_{80} b_{38} \text {, } \\
& b_{90}=b_{81} b_{39}, \quad b_{91}=b_{82} m_{3}, \quad b_{92}=b_{83} m_{4}, \quad b_{93}=b_{84} \overline{m_{3}}, \quad b_{94}=b_{85} \overline{m_{4}}, \\
& b_{95}=b_{61} e^{b_{5}}+b_{62} e^{b_{6}}+b_{63} e^{b_{7}}+b_{64} e^{b_{8}}+b_{65} e^{m_{1}}+b_{66} e^{m_{2}}+b_{67} e^{\overline{m_{1}}}+b_{68} e^{\overline{m_{2}}}+b_{69}, \\
& b_{96}=b_{78} e^{-b_{36}}+b_{79} e^{-b_{37}}+b_{80} e^{-b_{38}}+b_{81} e^{-b_{39}}+b_{82} e^{-m_{3}}+b_{83} e^{-m_{4}}+b_{84} e^{-\overline{m_{3}}}+b_{85} e^{\overline{-m_{4}}}-b_{86} \text {, } \\
& b_{97}=b_{61}+b_{62}+b_{63}+b_{64}+b_{65}+b_{66}+b_{67}+b_{68} \text {, } \\
& b_{98}=b_{78}+b_{79}+b_{80}+b_{81}+b_{82}+b_{83}+b_{84}+b_{85} \text {, } \\
& b_{99}=b_{70}+b_{71}+b_{72}+b_{73}+b_{74}+b_{75}+b_{76}+b_{77}+b_{69} \text {, } \\
& b_{100}=b_{87}+b_{88}+b_{89}+b_{90}+b_{91}+b_{92}+b_{93}+b_{94}+b_{86} \text {, } \\
& b_{101}=-e^{\lambda}+1, \quad b_{102}=-e^{-\lambda}+1, \quad b_{103}=-b_{96}+b_{98}+b_{95}-b_{97}, \quad b_{104}=\frac{\lambda}{\beta h}, \\
& b_{105}=\frac{b_{100}}{\beta h}-b_{99}, \quad b_{106}=\frac{b_{103} b_{104}-b_{105} b_{102}}{b_{101} b_{104}-\lambda b_{102}}, \quad b_{107}=\frac{-b_{105}+b_{106} \lambda}{b_{104}}, \quad d_{1}=-d_{2} e^{\lambda}-b_{95} \text {, } \\
& d_{3}=-d_{4} e^{-\lambda}-b_{96}, \quad d_{2}=b_{106}, \quad d_{4}=b_{107}, \quad b_{108}=\varepsilon \cos \omega t, \quad b_{109}=\lambda \varepsilon \cos \omega t, \\
& b_{110}=\varepsilon \omega \sin \omega t, \quad b_{111}=\varepsilon^{2} \cos ^{2} \omega t, \quad b_{112}=M^{2} \varepsilon^{2} \cos ^{2} \omega t, \quad b_{113}=M^{2} \operatorname{Re} \varepsilon \cos \omega t, \\
& b_{114}=M^{2} \operatorname{Re} \varepsilon \cos \omega t, \quad b_{115}=m_{5}+\overline{m_{5}}, \quad b_{116}=m_{5}+\overline{m_{6}}, \quad b_{117}=m_{6}+\overline{m_{5}},
\end{aligned}
$$


$b_{118}=m_{6}+\overline{m_{6}}, \quad b_{119}=b_{111} c_{5} m_{5} \overline{c_{5}} \overline{m_{5}}, \quad b_{120}=b_{111} c_{5} m_{5} \overline{c_{6}} \overline{m_{6}}, \quad b_{121}=b_{111} c_{6} m_{6} \overline{c_{5}} \overline{m_{5}}$,

$b_{122}=b_{111} c_{6} m_{6} \overline{c_{6}} m_{6}, \quad b_{123}=b_{112} c_{5} \overline{c_{5}}, \quad b_{124}=b_{112} c_{5} \overline{c_{6}}, \quad b_{125}=b_{112} c_{6} \overline{c_{5}}$,

$b_{126}=b_{112} \overline{c_{6}} \overline{c_{6}}, \quad b_{127}=b_{113} c_{5}, \quad b_{128}=b_{113} c_{6}, \quad b_{129}=b_{114} \overline{c_{5}}, \quad b_{130}=b_{114} \overline{c_{6}}$,

$b_{131}=b_{119}+b_{123}, \quad b_{132}=b_{120}+b_{124}, \quad b_{133}=b_{121}+b_{125}, \quad b_{134}=b_{122}+b_{126}, \quad b_{135}=\frac{b_{109}}{b_{108}}$,

$b_{136}=\frac{b_{110}}{b_{108}}, \quad b_{137}=\frac{b_{131}}{b_{108}}, \quad b_{138}=\frac{b_{132}}{b_{108}}, \quad b_{139}=\frac{b_{133}}{b_{108}}, \quad b_{139}=\frac{b_{133}}{b_{108}}, \quad b_{140}=\frac{b_{134}}{b_{108}}$,

$b_{141}=\frac{b_{127}}{b_{108}}, \quad b_{142}=\frac{b_{128}}{b_{108}}, \quad b_{143}=\frac{b_{129}}{b_{108}}, \quad b_{144}=\frac{b_{130}}{b_{108}}, \quad b_{145}=\varepsilon \cos \omega t, \quad b_{146}=K \varepsilon \cos \omega t$,

$b_{147}=\varepsilon \omega \sin \omega t, \quad b_{148}=b_{30} \varepsilon^{2} \cos ^{2} \omega t, \quad b_{149}=b_{31} \varepsilon^{2} \cos ^{2} \omega t, \quad b_{150}=b_{31} \operatorname{Re} \varepsilon \cos \omega t$,

$b_{151}=b_{31} \operatorname{Re} \varepsilon \cos \omega t, \quad b_{152}=\frac{b_{146}}{b_{145}}, \quad b_{153}=\frac{b_{147}}{b_{145}}, \quad b_{154}=\frac{b_{148}}{b_{145}}, \quad b_{156}=\frac{b_{150}}{b_{145}}, \quad b_{157}=\frac{b_{151}}{b_{145}}$,

$b_{158}=b_{154} c_{7} m_{7} \overline{c_{7}} \overline{m_{7}}, \quad b_{159}=b_{154} c_{7} m_{7} \overline{c_{8}} \overline{m_{8}}, \quad b_{160}=b_{154} c_{8} m_{8} \overline{c_{7}} \overline{m_{7}}, \quad b_{161}=b_{154} c_{8} m_{8} \overline{c_{8}} \overline{m_{8}}$,

$b_{162}=m_{7}+\overline{m_{7}}, \quad b_{163}=m_{7}+\overline{m_{8}}, \quad b_{164}=m_{8}+\overline{m_{7}}, \quad b_{165}=m_{8}+\overline{m_{8}}, \quad b_{166}=b_{155} c_{7} \overline{c_{7}}$,

$b_{167}=b_{155} c_{7} \overline{c_{8}}, \quad b_{168}=b_{155} c_{8} \overline{c_{7}}, \quad b_{169}=b_{155} c_{8} \overline{c_{8}}, \quad b_{170}=b_{156} c_{7}, \quad b_{171}=b_{156} c_{8}$,

$b_{172}=b_{157} \overline{c_{7}}, \quad b_{173}=b_{157} \overline{c_{8}}, \quad b_{174}=b_{158}+b_{166}, \quad b_{175}=b_{159}+b_{167}, \quad b_{176}=b_{160}+b_{168}$,

$b_{177}=b_{161}+b_{169}, \quad m_{9}=\frac{b_{135}+\sqrt{b_{135}^{2}-4 b_{136}}}{2}, \quad m_{10}=\frac{b_{135}-\sqrt{b_{135}^{2}-4 b_{136}}}{2}$,

$m_{11}=\frac{b_{152}+\sqrt{b_{152}^{2}-4 b_{153}}}{2}, \quad m_{12}=\frac{b_{152}-\sqrt{b_{152}^{2}-4 b_{153}}}{2}, \quad b_{178}=\frac{-b_{137}}{b_{115}^{2}-b_{135} b_{115}+b_{136}}$,

$b_{179}=\frac{-b_{138}}{b_{116}{ }^{2}-b_{135} b_{116}+b_{136}}, \quad b_{180}=\frac{-b_{139}}{b_{117}{ }^{2}-b_{135} b_{117}+b_{136}}, b_{181}=\frac{-b_{140}}{b_{118}^{2}-b_{135} b_{118}+b_{136}}$,

$b_{182}=\frac{-b_{141}}{m_{5}^{2}-b_{135} m_{5}+b_{136}}, \quad b_{183}=\frac{-b_{142}}{m_{6}^{2}-b_{135} m_{6}+b_{136}}, \quad b_{184}=\frac{-b_{143}}{{\overline{m_{5}}}^{2}-b_{135} \overline{m_{5}}+b_{136}}$, 


$$
\begin{aligned}
& b_{185}=\frac{-b_{144}}{{\overline{m_{6}}}^{2}-b_{135} \overline{m_{6}}+b_{136}}, \quad b_{186}=b_{178} b_{115}, \quad b_{187}=b_{179} b_{116}, \quad b_{188}=b_{180} b_{117}, \\
& b_{189}=b_{181} b_{118}, \quad b_{190}=b_{182} m_{5}, \quad b_{191}=b_{183} m_{6}, \quad b_{192}=b_{184} \overline{m_{5}}, \quad b_{193}=b_{185} \overline{m_{6}}, \\
& b_{194}=\frac{-b_{174}}{{b_{162}}^{2}-b_{152} b_{162}+b_{153}}, \quad b_{195}=\frac{-b_{175}}{b_{163}^{2}-b_{152} b_{163}+b_{153}}, \quad b_{196}=\frac{-b_{176}}{b_{164}^{2}-b_{152} b_{164}+b_{153}}, \\
& b_{197}=\frac{-b_{177}}{{b_{165}}^{2}-b_{152} b_{165}+b_{153}}, \quad b_{198}=\frac{-b_{170}}{m_{7}^{2}-b_{152} m_{7}+b_{153}}, \quad b_{199}=\frac{-b_{171}}{m_{8}^{2}-b_{152} m_{8}+b_{153}}, \\
& b_{200}=\frac{-b_{172}}{{\overline{m_{7}}}^{2}-b_{152} \overline{m_{7}}+b_{153}}, \quad b_{201}=\frac{-b_{173}}{{\overline{m_{8}}}^{2}-b_{152} \overline{m_{8}}+b_{153}}, \\
& b_{202}=b_{162} b_{194}, \quad b_{203}=b_{163} b_{195}, \quad b_{204}=b_{164} b_{196}, \quad b_{205}=b_{197} b_{165}, \quad b_{206}=b_{198} m_{7}, \\
& b_{207}=b_{199} m_{8}, \quad b_{208}=b_{200} \overline{m_{7}}, \quad b_{209}=b_{201} \overline{m_{8}}, \\
& b_{210}=b_{178} e^{b_{115}}+b_{179} e^{b_{116}}+b_{180} e^{b_{117}}+b_{181} e^{b_{118}}+b_{182} e^{m_{5}}+b_{183} e^{m_{6}}+b_{184} e^{\overline{m_{5}}}+b_{185} e^{\overline{m_{6}}}, \\
& b_{211}=b_{194} e^{-b_{162}}+b_{195} e^{-b_{163}}+b_{196} e^{-b_{164}}+b_{197} e^{-b_{165}}+b_{198} e^{-m_{7}}+ \\
& +b_{199} e^{-m_{8}}+b_{200} e^{-\overline{m_{7}}}+b_{201} e^{\overline{-m_{8}}} \text {, } \\
& b_{212}=b_{178}+b_{179}+b_{180}+b_{181}+b_{182}+b_{183}+b_{184}+b_{185} \text {, } \\
& b_{213}=b_{194}+b_{195}+b_{196}+b_{197}+b_{198}+b_{199}+b_{200}+b_{201} \text {, } \\
& b_{214}=b_{186}+b_{187}+b_{188}+b_{189}+b_{190}+b_{191}+b_{192}+b_{193} \text {, } \\
& b_{215}=b_{202}+b_{203}+b_{204}+b_{205}+b_{206}+b_{207}+b_{208}+b_{209} \text {, } \\
& b_{216}=m_{10}-m_{9}, \quad b_{217}=-b_{210} e^{-m_{9}}, \quad b_{218}=-m_{12}+m_{11}, \quad b_{219}=-b_{211} e^{m_{11}}, \\
& b_{220}=-e^{b_{216}}+1, \quad b_{221}=e^{b_{218}}-1, \quad b_{222}=b_{213}-b_{212}-b_{217}+b_{219}, \quad b_{223}=-m_{9} e^{b_{216}}+m_{10}, \\
& b_{224}=-\frac{m_{11}}{\beta h} e^{b_{218}}+\frac{m_{12}}{\beta h}, \quad b_{225}=-\frac{b_{215}}{\beta h}+\frac{b_{219} m_{11}}{\beta h}-m_{9} b_{217}-b_{214}, \\
& b_{226}=\frac{b_{222} b_{224}+b_{225} b_{221}}{b_{220} b_{224}+b_{223} b_{221}}, \quad b_{227}=-\left(\frac{b_{225}-b_{226} b_{223}}{b_{224}}\right), \quad d_{6}=b_{226}, \quad d_{5}=-d_{6} e^{b_{216}}+b_{217}, \\
& d_{7}=-d_{8} e^{b_{218}}+b_{219}, \quad d_{8}=b_{227} .
\end{aligned}
$$




\section{References}

[1] Shail R. (1973): On laminar tow-phase flow in magnetohydrodynamics. - International Journal of Engineering Science, vol.11, 1103.

[2] Walin G. (1969): Some aspects of time dependent motion of a stratified rotating fluid. - Journal of Fluid Mechanics, vol.36, 289.

[3] Packham B.A. and Shail R. (1971). Stratified laminar flow of two immiscible fluids. - Proceedings of Cambridge Philosophical Society, vol.69, pp.443-448.

[4] Lielausis O. (1975): Liquid metal magnetohydrodynamics. - Atomic Energy Review, vol.13, 527.

[5] Debnath L. and Basu U. (1975): Unsteady slip flow in an electrically conducting two-phase fluid under transverse magnetic fields. - NUOVO Cimento, vol.28B, pp.349-362.

[6] Michiyoshi Funakawa Kuramoto C., Akita Y. and Takahashi O. (1977): Instead of the helium-lithium annular-mist flow at high temperature, an air-mercury stratified flow in a horizontal rectangular duct in a vertical magnetic field. - Int. J. Multiphase Flow, vol.3, p.445.

[7] Dunn P.F. (1980): Single-Phase and Two-Phase Magnetohydrodynamic Pipe Flow. - International Journal of Heat Mass Transfer, vol.23, 373.

[8] Gherson P. and Lykoudis P.S. (1984): Local measurements in two-phase liquid-metal magneto-fluid mechanic flow. - Journal of Fluid Mechanics, vol.147, pp.81-104.

[9] Lohrasbi J. and Sahai V. (1989): Magnetohydrodynamic heat transfer in two-phase flow between parallel plates. Applied Scientific Research, vol.45, pp.53-66.

[10] Alireza S. and Sahai V. (1990): Heat transfer in developing magnetohydrodynamic Poiseuille flow and variable transport properties. - International Journal of Heat Mass Transfer, vol.33, No.8, pp.1711-1720.

[11] Serizawa A., Ida T., Takahashi O. and Michiyoshi I. (1990): MHD effect on Nak-nitrogen two-phase flow and heat transfer in a vertical round tube. - International Journal Multi-Phase Flow, vol.16, No.5, p.761.

[12] Malashetty M.S. and Leela V. (1992): Magnetohydrodynamic heat transfer in two phase flow. - International Journal of Engineering Science, vol.30, pp.371-377.

[13] Ramadan H.M. and Chamkha A.J. (1999): Two-phase free convection flow over an infinite permeable inclined plate with non-uniform particle-phase density. - International Journal of Engineering Science, vol.37, 1351.

[14] Chamkha A.J. (2000): Flow of two-immiscible fluids in porous and non-porous channels. - ASME Journal of Fluids Engineering, vol.122, pp.117-124.

[15] Raju T.L. and Murty P.S.R. (2006): Hydromagnetic two-phase flow and heat transfer through two parallel plates in a rotating system. - Journal of Indian Academy of Mathematics, Indore, India, vol.28, No.2, pp.343-360.

[16] Tsuyoshi I. and Shu-Ichiro I. (2008): Two-fluid magnetohydrodynamic simulation of converging Hi flows in the interstellar medium. - The Astrophysical Journal, vol.687, No.1, pp.303-310.

[17] Haim H.B., Jianzhong Z., Shizhi Q. and X. Yu X. (2003): A magneto-hydrodynamically controlled fluidic network. - Sensors and Actuators B, vol.88, No.2, pp.205-216.

[18] Hussameddine S.K., Martin J.M. and Sang W.J. (2008): Analytical prediction of flow field in magnetohydrodynamic-based microfluidic devices. - Journal of Fluids Engineering, vol.130, No.9, 6.

[19] Yi M., Qian S. and Bau H. (2002): A magnetohydrodynamic chaotic stirrer. - Journal of Fluid Mechanics, vol.468, pp.153-177.

[20] Weston M.C., Gerner M.D. and Fritsch I. (2010): Magnetic fields for fluid motion. - Analytical Chemistry, vol.82, No.9, pp.3411-3418.

[21] Hide R. and Roberts P.H. (1961): The origin of the mean geomagnetic field. - In: Physics and Chemistry of the Earth. Pergamon Press, New York, vol.4, pp.27-98 . 
[22] Greenspan H.P. and Howard L.N. (1963): On a time dependent motion of a rotating fluid. - Journal of Fluid Mechanics, vol.17, p.385.

[23] Dieke R.H. (1970): Internal rotation of the sun. - In: L. Goldberg (eds.), Annual Reviews of Astronomy and Astrophysics, vol.8, Annual Reviews Inc., pp.297-328.

[24] Elco R.A., Hughes W.F. and Young F.J. (1962): Theoretical analysis of the radial filed vortex magneto-gas dynamic generator. - Zeitschrift fur Angewandte Mathematik and Physik (ZAMP), vol.13, pp.1-13.

[25] Katsurai M. (1972): Studies on the MHD rotating machine. - Electrical Engineering in Japan, vol.92, pp.31-43.

[26] Kolesnikov V.K. and Khait V.D. (1975): Nonlinear fluctuations in a MHD generator. - Teplofizika Vysokikh Temperatur, vol.13, pp.601-604.

[27] Yantovskiy Y.I. and Tolmach I.M. (1963): On the theory of an asynchronous magnetohydrodynamic generator with a rotating field. - Technical Report, Wright-Patterson Air Force Base, Ohio, USA.

[28] Holton J.R. (1965): The influence of viscous boundary layers on transient motions in a stratified rotating fluid. International Journal of Atmospheric Science, vol.22, p.402.

[29] Batchlor G.K. (1967): An Introduction to Fluid Dynamics. - I Edition, Cambridge press, Cambridge, UK.

[30] Gupta A.S. (1972): Magnetohydrodynamic Ekmann layer. - Acta Mechanica, vol.13, pp.155.

[31] Tao L.N. (1960): Magnetohydrodynamic effects on the formation of Couette flow. - Journal of Aero/Space Science, vol.27, pp.334-338.

[32] Gupta A.S. (1960): On the flow of an electrically conducting fluid near an accelerated plate in the presence of a magnetic field. - J. Phys. Soc. Japan, vol.15, No.10, pp.1894-1897.

[33] Stanisic M.M., Fetz B.H., Mickelsen Jr. H.P. and Czumak F.M. (1962): On the flow of a hydromagnetic fluid between two oscillating flat plates. - Journal of Aero/Space Science, vol.29, No.1, pp.116-117.

[34] Katagiri M. (1962): Flow formation in Couette motion in magnetohydrodynamcis. - J. Phys. Soc. Japan, vol.17, No.2, pp.393-396.

[35] Nanda R.S. and Mohanty H.K. (1971): Hydromagnetic flow in a rotating channel. - Applied Scientific Research, vol.24, p.65.

[36] Debnath L. (1972): On unsteady magnetohydrodynamic boundary layers in a rotating system. - ZAMM., vol.52, p.623.

[37] Jana R.N., Datta N. and Mazumder B.S. (1977): Magnetohydrodynamic Couette flow and heat transfer in a rotating system. - Journal of the Physical Society of Japan, vol.42, p.1034.

[38] Seth G.S., Jana R.N. and Maiti M.K. (1982): Unsteady hydromagnetic Couette flow in a rotating system. International Journal of Engineering Science, vol.20, p.989.

[39] Ghosh S.K. (1993): Unsteady hydromagnetic flow in a rotating channel with oscillating pressure gradient. Journal of the Physical Society of Japan, vol.62, p.3893.

[40] Pallath Chandran, Nirmal C. Sacheti and Ashok K. Singh (1998): Unsteady hydromagnetic free convection flow with heat flux and accelerated boundary motion. - J. Phys. Soc. Japan, vol.67, p.124.

[41] Ghosh S.K. and Bhattacharjee P.K. (2000): Magnetohydrodynamic convective flow in a rotating channel. Archives of Mechanics, vol.52, p.303.

[42] Singh K.D. (2000): An oscillatory hydromagnetic Couette flow in a rotating system. - ZAMM, vol.80, p.429.

[43] Hayat T., Nadeem S. and Asghar S. (2004): Hydromagnetic Couette flow of an Oldroyd-B fluid in a rotating system. - International Journal of Engineering Science, vol.42, p.65.

[44] Guria M. and Jana R.N. (2007): Hydromagnetic flow in the Ekman layer on an oscillating porous plate. Magnetohydrodynamics, vol.43, pp.3-11.

[45] Chamkha A.J. (2004): Unsteady MHD convective heat and mass transfer past a semi-infinite vertical permeable moving plate with heat absorption. - International Journal of Engineering Science, vol.42, pp.217-230. 
[46] Umavathi J.C., Abdul Mateen, Chamkha A.J. and Al- Mudhaf A. (2006): Oscillatory Hartmann two-fluid flow and heat transfer in a horizontal channel. - International Journal of Applied Mechanics and Engineering, vol.11, No.1, pp.155-178.

[47] Raju T.L. and Sreedhar S. (2009). Unsteady two-fluid flow and heat transfer of conducting fluids in channels under transverse magnetic field. - International Journal of Applied Mechanics and Engineering, vol.14, No.4, pp.1093-1114.

[48] Raju T.L. and Rao B.N. (2014): Unsteady two-layered fluid flow of conducting fluids in a channel between parallel porous plates under transverse magnetic field in a rotating system. - Communicated for favour of publication in International Journal of Applied Mechanics and Engineering.

Received: September 10, 2016

Revised: April 12, 2016 